\title{
Uniform Bahadur Representation for Local Polynomial Estimates of M-Regression and Its Application to The Additive Model
}

\author{
Efang Kong* \\ (Technische Universiteit Eindhoven) \\ Oliver Linton $^{\dagger}$ \\ (London School of Economics) \\ Yingcun $\mathrm{Xia}^{\ddagger}$ \\ (National University of Singapore)
}

DP No: EM 2009535

The Suntory Centre

Suntory and Toyota International Centres for

Economics and Related Disciplines

London School of Economics and Political Science Houghton Street

London WC2A 2AE

2009

Tel: 02079556674

\footnotetext{
*Eurandom, Technische Universiteit Eindhoven, The Netherlands. E-mail address: kong@eurandom.tue.nl.

Department of Economics, London School of Economics, Houghton Street, London WC2A 2AE, United Kingdom. http://econ.Ise.ac.uk/staff/olinton/index own.html. E-mail address: o.linton@lse.ac.uk.

$\ddagger$ Department of Statistics and Applied Probability, National University of Singapore, Singapore. http://www.stat.nus.edu.sg/ staxyc. E-mail address: staxyc@nus.edu.sg.
} 


\begin{abstract}
We use local polynomial fitting to estimate the nonparametric M-regression function for strongly mixing stationary processes $\left\{\left(Y_{i}, \underline{X}_{i}\right)\right\}$. We establish a strong uniform consistency rate for the Bahadur representation of estimators of the regression function and its derivatives. These results are fundamental for statistical inference and for applications that involve plugging such estimators into other functional where some control over higher order terms are required. We apply our results to the estimation of an additive M-regression model.
\end{abstract}

(c) The authors. All rights reserved. Short sections of text, not to exceed two paragraphs, may be quoted without explicit permission provided that full credit, including $\odot$ notice, is given to the source. 


\title{
Uniform Bahadur Representation for Local Polynomial Estimates of M-Regression and Its Application to The Additive Model
}

\author{
EFANG KONG* \\ Technische Universiteit Eindhoven \\ OLIVER LINTON ${ }^{\dagger}$ \\ London School of Economics \\ YINGCUN XIA ${ }^{\ddagger}$ \\ National University of Singapore
}

We use local polynomial fitting to estimate the nonparametric M-regression function for strongly mixing stationary processes $\left\{\left(Y_{i}, \underline{X}_{i}\right)\right\}$. We establish a strong uniform consistency rate for the Bahadur representation of estimators of the regression function and its derivatives. These results are fundamental for statistical inference and for applications that involve plugging such estimators into other functionals where some control over higher order terms are required. We apply our results to the estimation of an additive M-regression model.

\section{INTRODUCTION}

In many contexts one wants to evaluate the properties of some procedure that is a functional of some given estimators. It is useful to be able to work with some plausible high level assumptions about those estimators rather than to re-derive their properties for each different application. In a fully parametric (and stationary, weakly dependent data) context, it is quite common to assume that estimators are root-n consistent and asymptotically normal. In some cases this property suffices; in other cases one needs to be more explicit in terms of the linear expansion of these estimators, but in any case such expansions are quite natural and widely applicable. In

\footnotetext{
*Eurandom, Technische Universiteit Eindhoven, The Netherlands. E-mail address: kong@eurandom.tue.nl.

${ }^{\dagger}$ Department of Economics, London School of Economics, Houghton Street, London WC2A 2AE, United Kingdom. http://econ.lse.ac.uk/staff/olinton/ index_own.html. E-mail address: o.linton@lse.ac.uk.

${ }^{\ddagger}$ Department of Statistics and Applied Probability, National University of Singapore, Singapore. http://www.stat.nus.edu.sg/ staxyc. E-mail address: staxyc@nus.edu.sg.
} 
a nonparametric context there is less agreement about the use of such expansions and one often sees standard properties of standard estimators derived anew for a different purpose. It is our objective to provide results that can circumvent this. The types of application we have in mind are estimation of semiparametric models where the parameters of interest are explicit or implicit functionals of nonparametric regression functions and their derivatives; see Powell (1994), Andrews (1994) and Chen, Linton and Van Keilegom (2003). Another class of applications includes estimation of structured nonparametric models like the additive models (Linton and Nielsen, 1995) or the generalized additive models (Linton, Sperlich and Van Keilegom, 2007).

We motivate our results in a simple i.i.d. setting. Suppose we have a random sample $\left\{Y_{i}, X_{i}\right\}_{i=1}^{n}$ and consider the Nadaraya-Watson estimator of the regression function $m(x)=$ $E\left(Y_{i} \mid X_{i}=x\right)$,

$$
\hat{m}(x)=\frac{\hat{r}(x)}{\hat{f}(x)}=\frac{n^{-1} \sum_{i=1}^{n} K_{h}\left(X_{i}-x\right) Y_{i}}{n^{-1} \sum_{i=1}^{n} K_{h}\left(X_{i}-x\right)},
$$

where $K($.$) is a symmetric density function, h$ is a bandwidth and $K_{h}()=.K(. / h) / h$. Standard arguments (Härdle, 1990) show that under suitable smoothness conditions,

$$
\hat{m}(x)-m(x)=h^{2} b(x)+\frac{1}{n f(x)} \sum_{i=1}^{n} K_{h}\left(X_{i}-x\right) \varepsilon_{i}+R_{n}(x),
$$

where $b(x)=\int u^{2} K(u) d u\left[m^{\prime \prime}(x)+2 m^{\prime}(x) f^{\prime}(x) / f(x)\right] / 2$, while $f(x)$ is the covariate density function and $\varepsilon_{i} \equiv Y_{i}-m\left(X_{i}\right)$ is the error term. The remainder term $R_{n}(x)$ is of smaller order (almost surely) than the two leading terms. Such an expansion is sufficient to derive the central limit theorem for $\hat{m}(x)$ itself, but generally is not sufficient if $\hat{m}(x)$ is to be plugged into some semiparametric procedure. For example, suppose we estimate the parameter $\theta_{0}=\int m(x)^{2} d x \neq 0$ by $\hat{\theta}=\int \hat{m}(x)^{2} d x$, where the integral is over some compact set $\mathcal{D}$; we would expect to find that $n^{1 / 2}\left(\hat{\theta}-\theta_{0}\right)$ is asymptotically normal. Based on expansion (1), the argument goes like this. First, we obtain the following

$$
n^{1 / 2}\left(\hat{\theta}-\theta_{0}\right)=2 n^{1 / 2} \int m(x)\{\hat{m}(x)-m(x)\} d x+n^{1 / 2} \int[\hat{m}(x)-m(x)]^{2} d x .
$$

If it can be shown that $\hat{m}(x)-m(x)=o\left(n^{-1 / 4}\right)$ a.s. uniformly in $x \in \mathcal{D}$ ( such results are widely 
available; see for example Masry (1996)), we have

$$
n^{1 / 2}\left(\hat{\theta}-\theta_{0}\right)=2 n^{1 / 2} \int m(x)\{\hat{m}(x)-m(x)\} d x+o(1) \quad \text { a.s. }
$$

Note that the quantity on the right hand side is the term in assumption 2.6 of Chen, Linton, and Van Keilegom (2003) which is assumed to be asymptotically normal. It is the verification of this condition with which we are now concerned. We substitute in expansion (1) and obtain

$$
\begin{gathered}
n^{1 / 2}\left(\hat{\theta}-\theta_{0}\right)=2 n^{1 / 2} h^{2} \int m(x) b(x) d x+2 n^{1 / 2} \int n^{-1} \sum_{i=1}^{n} \varepsilon_{i} K_{h}\left(X_{i}-x\right) \frac{m(x)}{f(x)} d x \\
+2 n^{1 / 2} \int m(x) R_{n}(x) d x+o(1) \quad \text { a.s. }
\end{gathered}
$$

If $n h^{4} \rightarrow 0$, then the first term (the smoothing bias term) is $o(1)$. The second term (the stochastic term) is a sum of independent random variables with mean zero, which can be rewritten, using a change of variables, as

$$
\begin{gathered}
n^{1 / 2} \int m(x) f^{-1}(x) n^{-1} \sum_{i=1}^{n} K_{h}\left(X_{i}-x\right) \varepsilon_{i} d x=n^{-1 / 2} \sum_{i=1}^{n} \xi_{n}\left(X_{i}\right) \varepsilon_{i} \\
\xi_{n}\left(X_{i}\right)=\int m\left(X_{i}+u h\right) f^{-1}\left(X_{i}+u h\right) K(u) d u
\end{gathered}
$$

and this term obeys the Lindeberg central limit theorem under standard conditions. The problem is with the third term, as equation (1) only guarantees that $\int m(x) R_{n}(x) d x=o\left(n^{-2 / 5}\right)$ a.s. at best. In fact, it is possible to derive a more useful Bahadur representation (Bahadur, 1966) for the kernel estimator

$$
\hat{m}(x)-m(x)=h^{2} b_{n}(x)+\{E \hat{f}(x)\}^{-1} n^{-1} \sum_{i=1}^{n} K_{h}\left(X_{i}-x\right) \varepsilon_{i}+R_{n}^{*}(x),
$$

where $b_{n}(x)$ is deterministic and satisfies $b_{n}(x) \rightarrow b(x)$ and $E \hat{f}(x) \rightarrow f(x)$ uniformly in $x \in \mathcal{D}$, while the remainder term now satisfies

$$
\sup _{x \in \mathcal{D}}\left|R_{n}^{*}(x)\right|=O\left(\frac{\log n}{n h}\right) \quad \text { a.s. }
$$

This property is a consequence of the uniform convergence rate of $\hat{f}(x)-E \hat{f}(x), n^{-1} \sum_{i=1}^{n} K_{h}(x$ $\left.-X_{i}\right)\left\{m\left(X_{i}\right)-m(x)\right\}-E K_{h}\left(X_{i}-x\right)\left\{m\left(X_{i}\right)-m(x)\right\}$ and $n^{-1} \sum_{i=1}^{n} K_{h}\left(X_{i}-x\right) \varepsilon_{i}$ that follow 
from, for example Masry (1996). Clearly, by appropriate choice of the bandwidth $h, R_{n}^{*}(x)$ can be made $o\left(n^{-1 / 2}\right)$ a.s. uniformly over $\mathcal{D}$ and thus $2 n^{1 / 2} \int m(x) R_{n}^{*}(x) d x=o(1)$ a.s.. Therefore, to derive asymptotic normality for $n^{1 / 2}\left(\hat{\theta}-\theta_{0}\right)$, one can just work with the two leading terms in (2). These terms are slightly more complicated than in the previous expansion but are still sufficiently simple for many purposes; in particular, $b_{n}(x)$ is uniformly bounded so that provided $n h^{4} \rightarrow 0$, the smoothing bias term satisfies $h^{2} n^{1 / 2} \int m(x) b_{n}(x) d x \rightarrow 0$, while the stochastic term is a sum of zero mean independent random variables

$$
\begin{gathered}
n^{1 / 2} \int \frac{m(x)}{\bar{f}(x)} n^{-1} \sum_{i=1}^{n} K_{h}\left(X_{i}-x\right) \varepsilon_{i} d x=n^{-1 / 2} \sum_{i=1}^{n} \bar{\xi}_{n}\left(X_{i}\right) \varepsilon_{i} \\
\bar{\xi}_{n}\left(X_{i}\right)=\int \frac{m\left(X_{i}+u h\right)}{\bar{f}\left(X_{i}+u h\right)} K(u) d u
\end{gathered}
$$

and obeys the Lindeberg central limit theorem under standard conditions, where $\bar{f}(x)=E \hat{f}(x)$. This argument shows the utility of Bahadur representation (2). There are many other applications of this result because a host of probabilistic results are available for random variables like $n^{-1} \sum_{i=1}^{n} K_{h}\left(X_{i}-x\right) \varepsilon_{i}$ and integrals thereof.

The one-dimensional Nadaraya-Watson estimator for i.i.d. data is particularly easy to analyze and the above arguments are well known. However, the limitations of this estimator are manyfold and there are good theoretical reasons for working instead with the local polynomial class of estimators (Fan and Gijbels, 1996). In addition, for many data especially financial time series data one may have concerns about heavy tails or outliers that point in the direction of using robust estimators like the local median or local quantile method, perhaps combined with local polynomial fitting. We examine a general class of (nonlinear) M-regression function (that is, location functionals defined through minimization of a general objective function $\rho()$.$) and$ derivative estimators. We treat a general time series setting where the multivariate data are strongly mixing. Under mild conditions, we establish a uniform strong Bahadur representation like (2) and (3) with remainder term of order $\left(\log n / n h^{d}\right)^{3 / 4}$ almost surely, a rate that is almost optimal or in other words can't be improved further based on the results in Kiefer (1967) under i.i.d. setting. The leading terms are linear and functionals of them can be analyzed simply. 
The remainder term can be made to be $o\left(n^{-1 / 2}\right)$ a.s. under restrictions on the dimensionality in relation to the amount of smoothness possessed by the M-regression function.

The best convergence rate of unrestricted nonparametric estimators strongly depends on $d$, the dimension of the covariates. The rate decreases dramatically as $d$ increases (Stone, 1982). This phenomenon is the so-called "curse of dimensionality". One approach to reduce the curse is by imposing model structure. A popular model structure is the additive model assuming that

$$
m\left(x_{1}, \ldots, x_{d}\right)=c+m_{1}\left(x_{1}\right)+\ldots+m_{d}\left(x_{d}\right)
$$

where $c$ is an unknown constant and $m_{k}(),. k=1, \ldots, d$ are unknown functions which have been normalized such that $\operatorname{Em}_{k}\left(\mathbf{x}_{k}\right)=0, k=1, \ldots, d$. In this case, the optimal rate of convergence is the same as in univariate nonparametric regression (Stone, 1986). An additive M-regression function is given by (4), where $m(x)$ is the M-regression function defined in (5) for some loss function $\rho(. ;$.$) . Previous work on additive quantile regression, for example, includes Linton$ (2001) and Horowitz and Lee (2005) for the i.i.d. case. An interesting application of the additive M-regression model is to combine (4) with the volatility model

$$
Y_{i}=\sigma_{i} \varepsilon_{i} \quad \text { and } \quad \ln \sigma_{i}^{2}=m\left(X_{i}\right)
$$

where $X_{i}=\left(Y_{i-1}, \ldots, Y_{i-d}\right)^{\top}$. We suppose that $\varepsilon_{i}$ satisfies $E\left[\varphi\left(\ln \varepsilon_{i}^{2} ; 0\right) \mid X_{i}\right]=0$ with $\varphi(. ;$.$) the$ piecewise derivative of $\rho(. ;$.$) , whence m($.$) is the conditional M$-regression of $\ln Y_{i}^{2}$ given $X_{i}$. Peng and Yao (2003) applied LAD estimation to parametric ARCH and GARCH models and showed the superior robustness property of this procedure over Gaussian QMLE with regard to heavy tailed innovations. This heavy tail issue also arises in nonparametric regression models and empirical evidences suggest that moderately high frequency financial data are often heavy tailed, which is why our procedures may be useful. We apply the Bahadur representations to the study of the marginal integration estimators (Linton and Nielsen, 1995) of the component functions in the additive M-regression model in which case we only need the remainder term to be $o\left(n^{-p /(2 p+1)}\right)$ a.s., where $p$ is a smoothness index.

Bahadur representations (Bahadur, 1966) have been widely studied and applied, with notable 
refinements in the i.i.d. setting by Kiefer (1967). A recent paper of Wu (2005) extends these results to a general class of dependent processes and provides a review. The closest paper to ours is Hong (2003), which established the Bahadur representation for essentially the same local polynomial M-regression estimator as ours. However, his results are: (a) pointwise, i.e., for a single $x$ only; (b) the covariate is univariate; (c) for i.i.d. data. Clearly, this limits the range of applicability of his results, and specifically, the applications to semiparametric or additive models are perforce precluded.

\section{THE GENERAL SETTING}

Let $\left\{\left(Y_{i}, \underline{X}_{i}\right)\right\}$ be a jointly stationary processes, where $\underline{X}_{i}=\left(\mathbf{x}_{i 1}, \ldots, \mathbf{x}_{i d}\right)^{\top}$ with $d \geq 1$ and $Y_{i}$ is a scalar. As dependent observations are considered in this paper, we introduce here the mixing coefficient. Let $\mathbf{F}_{s}^{t}$ be the $\sigma$ - algebra of events generated by random variables $\left\{\left(Y_{i}, \underline{X}_{i}\right), s \leq i \leq\right.$ $t\}$. A stationary stochastic processes $\left\{\left(Y_{i}, \underline{X}_{i}\right)\right\}$ is strongly mixing if

$$
\sup _{\substack{A \in \mathbf{F}^{0} \\ B \in \overline{\mathbf{F}}_{k}^{\infty}}}|P[A B]-P[A] P[B]|=\gamma[k] \rightarrow 0, \text { as } k \rightarrow \infty,
$$

and $\gamma[k]$ is called the strong mixing coefficient.

Suppose $\rho(. ;$.$) is a loss function. Our first goal is to estimate the multivariate M-regression$ function

$$
m\left(x_{1}, \cdots, x_{d}\right)=\arg \min _{\theta} E\left\{\rho\left(Y_{i} ; \theta\right) \mid \underline{X}_{i}=\left(x_{1}, \cdots, x_{d}\right)\right\}
$$

and its partial derivatives based on observations $\left\{\left(Y_{i}, \underline{X}_{i}\right)\right\}_{i=1}^{n}$. An important example of the M-function is the $q$ th $(0<q<1)$ quantile of $Y_{i}$ given $\underline{X}_{i}=\left(x_{1}, \cdots, x_{d}\right)^{\top}$, with loss function $\rho(y ; \theta)=(2 q-1)(y-\theta)+|y-\theta|$. Another example is the $L_{q}$ criterion: $\rho(y ; \theta)=|y-\theta|^{q}$ for $q>1$, which includes the least square criterion $\rho(y ; \theta)=(y-\theta)^{2}$ with $m($.$) the conditional expectation$ of $Y_{i}$ given $\underline{X}_{i}$. Yet another example is the celebrated Huber's function (Huber, 1973)

$$
\rho(t)=t^{2} / 2 I\{|t|<k\}+\left(k|t|-k^{2} / 2\right) I\{|t| \geq k\} .
$$


Suppose $m(\underline{x})$ is differentiable up to order $p+1$ at $\underline{x}=\left(x_{1}, \ldots, x_{d}\right)^{\top}$. Then the multivariate $p^{\prime}$ th order local polynomial approximation of $m(\underline{z})$ for any $\underline{z}$ close to $\underline{x}$ is given by

$$
m(\underline{z}) \approx \sum_{0 \leq|\underline{r}| \leq p} \frac{1}{r !} D^{\underline{r}} m(\underline{x})(\underline{z}-\underline{x})^{\underline{r}},
$$

where $\underline{r}=\left(r_{1}, \ldots, r_{d}\right),|\underline{r}|=\sum_{i=1}^{d} r_{i}, \underline{r} !=r_{1} ! \times \cdots \times r_{d} !$, and

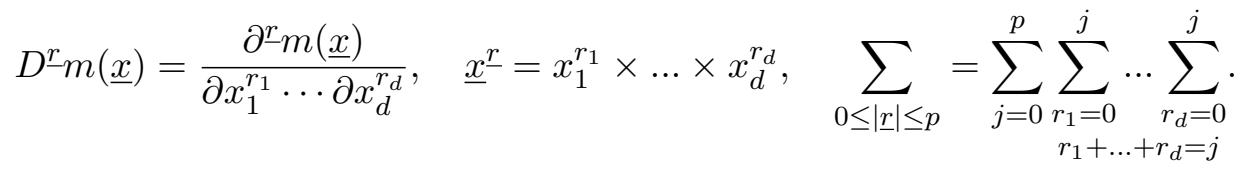

Let $K(\underline{u})$ be a density function on $R^{d}, h$ a bandwidth and $K_{h}(\underline{u})=K(\underline{u} / h)$. With observations $\left\{\left(Y_{i}, \underline{X}_{i}\right)\right\}_{i=1}^{n}$, we consider minimizing the following quantity with respect to $\beta_{\underline{r}}, 0 \leq|\underline{r}| \leq p$ :

$$
\sum_{i=1}^{n} K_{h}\left(\underline{X}_{i}-\underline{x}\right) \rho\left(Y_{i} ; \sum_{0 \leq|\underline{r}| \leq p} \beta_{\underline{r}}\left(\underline{X}_{i}-\underline{x}\right)^{\underline{r}}\right)
$$

Denote by $\hat{\beta}_{\underline{r}}(\underline{x}), 0 \leq|r| \leq p$, the minima of (8). The M-regression function $m(\underline{x})$ and its partial derivatives $D^{\underline{r}} m(\underline{x}), 1 \leq|\underline{r}| \leq p$ are then estimated respectively by

$$
\hat{m}(\underline{x})=\hat{\beta}_{\underline{0}}(\underline{x}) \quad \text { and } \quad \hat{D}^{\underline{r}} m(\underline{x})=\underline{r} ! \hat{\beta}_{\underline{r}}(\underline{x}), 1 \leq|\underline{r}| \leq p .
$$

\section{MAIN RESULTS}

In Theorem 3.2 below we give our main result, the uniform strong Bahadur representation for the vector $\hat{\beta}_{p}(\underline{x})$. We first need to develop some notations to define the leading terms in the representation.

Let $N_{i}=\left(\begin{array}{c}i+d-1 \\ d-1\end{array}\right)$ be the number of distinct $d$-tuples $\underline{r}$ with $|\underline{r}|=i$. Arrange these $d$-tuples as a sequence in a lexicographical order (with the highest priority given to the last position so that $(0, \cdots, 0, i)$ is the first element in the sequence and $(i, 0, \cdots, 0)$ the last element). Let $\tau_{i}$ denote this 1 -to- 1 mapping, i.e. $\tau_{i}(1)=(0, \cdots, 0, i), \cdots, \tau_{i}\left(N_{i}\right)=(i, 0, \cdots, 0)$. For each $i=1, \cdots, p$, define a $N_{i} \times 1$ vector $\mu_{i}(\underline{x})$ with its $k$ th element given by $\underline{x}^{\tau_{i}(k)}$ and write $\mu(\underline{x})=$ $\left(1, \mu_{1}(\underline{x})^{\top}, \cdots, \mu_{p}(\underline{x})^{\top}\right)^{\top}$, which is a column vector of length $N=\sum_{i=0}^{p} N_{i}$. Similarly define vectors $\beta_{p}(\underline{x})$ and $\underline{\beta}$ through the same lexicographical arrangement of $D^{\underline{r}} m(\underline{x})$ and $\beta_{\underline{r}}$ in (8) for 
$0 \leq|\underline{r}| \leq p$. Thus (8) can be rewritten as

$$
\sum_{i=1}^{n} K_{h}\left(\underline{X}_{i}-\underline{x}\right) \rho\left(Y_{i} ; \mu\left(\underline{X}_{i}-\underline{x}\right)^{\top} \underline{\beta}\right)
$$

Suppose the minimizer of $(10)$ is denoted as $\hat{\beta}_{n}(\underline{x})$. Let $\hat{\beta}_{p}(\underline{x})=W_{p} \hat{\beta}_{n}(\underline{x})$, where $W_{p}$ is a diagonal matrix with diagonal entries the lexicographical arrangement of $\underline{r} !, 0 \leq|\underline{r}| \leq p$.

Let $\nu_{i}=\int K(\underline{u}) \underline{u} \underline{i} d \underline{u}$. For $g($.$) given in (A.7), define$

$$
\nu_{n \underline{i}}(\underline{x})=\int K(\underline{u}) \underline{u}^{\underline{i}} g(\underline{x}+h \underline{u}) f(\underline{x}+h \underline{u}) d \underline{u} .
$$

For $0 \leq j, k \leq p$, let $S_{j, k}$ and $S_{n, j, k}(\underline{x})$ be two $N_{j} \times N_{k}$ matrices with their $(l, m)$ elements respectively given by

$$
\left[S_{j, k}\right]_{l, m}=\nu_{\tau_{j}(l)+\tau_{k}(m)}, \quad\left[S_{n, j, k}(\underline{x})\right]_{l, m}=\nu_{n, \tau_{j}(l)+\tau_{k}(m)}(\underline{x})
$$

Now define the $N \times N$ matrices $S_{p}$ and $S_{n, p}(\underline{x})$ by

$$
S_{p}=\left[\begin{array}{cccc}
S_{0,0} & S_{0,1} & \cdots & S_{0, p} \\
S_{1,0} & S_{1,1} & \cdots & S_{1, p} \\
\vdots & \ddots & \vdots & \\
S_{p, 0} & S_{p, 1} & \cdots & S_{p, p}
\end{array}\right], \quad S_{n, p}(\underline{x})=\left[\begin{array}{cccc}
S_{n, 0,0}(\underline{x}) & S_{n, 0,1}(\underline{x}) & \cdots & S_{n, 0, p}(\underline{x}) \\
S_{n, 1,0}(\underline{x}) & S_{n, 1,1}(\underline{x}) & \cdots & S_{n, 1, p}(\underline{x}) \\
\vdots & \ddots & \vdots & \\
S_{n, p, 0}(\underline{x}) & S_{n, p, 1}(\underline{x}) & \cdots & S_{n, p, p}(\underline{x})
\end{array}\right] .
$$

According to Lemma $5.8, S_{n, p}(\underline{x})$ converges to $g(\underline{x}) f(\underline{x}) S_{p}$ uniformly in $\underline{x} \in \mathcal{D}$ almost surely. Hence for $\left|S_{p}\right| \neq 0$, we can define

$$
\beta_{n}^{*}(\underline{x})=-\frac{1}{n h^{d}} W_{p} S_{n, p}^{-1}(\underline{x}) H_{n}^{-1} \sum_{i=1}^{n} K_{h}\left(\underline{X}_{i}-\underline{x}\right) \varphi\left(Y_{i}, \mu\left(\underline{X}_{i}-\underline{x}\right)^{\top} \beta_{p}(\underline{x})\right) \mu\left(\underline{X}_{i}-\underline{x}\right),
$$

where $\varphi(. ;$.$) is the piecewise derivative of \rho(.,$.$) as defined in (A1) and H_{n}$ is a diagonal matrix with diagonal entries $h^{|\underline{r}|}, 0 \leq|\underline{r}| \leq p$ in the aforementioned lexicographical order. The quantity $\beta_{n}^{*}(\underline{x})$ is the leading term in the Bahadur representation of $\hat{\beta}_{p}(\underline{x})-\beta_{p}(\underline{x})$; it is the sum of a bias term, $E \beta_{n}^{*}(\underline{x})$, and a stochastic term $\beta_{n}^{*}(\underline{x})-E \beta_{n}^{*}(\underline{x})$.

Denote the typical element of $\beta_{n}^{*}(\underline{x})$ by $\beta_{n \underline{r}}^{*}(\underline{x}), 0 \leq|\underline{r}| \leq p$ and the probability density function of $\underline{X}$ by $f($.$) . The following results on E \beta_{n \underline{r}}^{*}(\underline{x})$ is an extension of Proposition 2.2 in Hong (2003) to the multivariate case. 
PROPOSITION 3.1 If $f(\underline{x})>0$ and conditions (A1)-(A5) in the Appendix hold, then

$$
E \beta_{n \underline{r}}^{*}(\underline{x})=\left\{\begin{array}{lr}
-h^{p+1} e_{N(\underline{r})} W_{p} S_{p}^{-1} B_{1} \mathbf{m}_{p+1}(\underline{x})+o\left(h^{p+1}\right), & \text { for } p-|\underline{r}| \text { odd }, \\
-h^{p+2} e_{N(\underline{r})} W_{p} S_{p}^{-1}\left[\{f g\}^{-1}(\underline{x}) \mathbf{m}_{p+1}(\underline{x})\left\{\tilde{M}(\underline{x})-N_{p} S_{p}^{-1} B_{1}\right\}+B_{2} \mathbf{m}_{p+2}(\underline{x})\right] \\
+o\left(h^{p+2}\right), & \text { for } p-|\underline{r}| \text { even },
\end{array}\right.
$$

where $N(\underline{r})=\tau_{|\underline{r}|}^{-1}(\underline{r})+\sum_{k=0}^{|\underline{r}|-1} N_{k}, e_{i}$ is a $N \times 1$ vector having 1 as the ith entry with all other entries 0 , and $B_{1}=\left[S_{0, p+1}, S_{1, p+1}, \cdots S_{p, p+1}\right]^{\top}, B_{2}=\left[S_{0, p+2}, S_{1, p+2}, \cdots S_{p, p+2}\right]^{\top}$.

We next present our main result, the Bahadur representation for the local polynomial estimates $\hat{\beta}_{p}(\underline{x})$.

THEOREM 3.2 Suppose (A1)-(A7) in the Appendix hold with $\lambda_{2}=(p+1) / 2(p+s+1)$ for some $s \geq 0$ and $\mathcal{D}$ is any compact subset of $R^{d}$. Then

$$
\sup _{\underline{x} \in \mathcal{D}}\left|H_{n}\left\{\hat{\beta}_{p}(\underline{x})-\beta_{p}(\underline{x})\right\}-\beta_{n}^{*}(\underline{x})\right|=O\left(\left\{\frac{\log n}{n h^{d}}\right\}^{\lambda(s)}\right) \text { almost surely }
$$

where |.| is taken to be the sup norm and

$$
\lambda(s)=\min \left\{\frac{p+1}{p+s+1}, \frac{3 p+3+2 s}{4 p+4 s+4}\right\} .
$$

REMARK 1. According to Theorem 1 in Kiefer (1967), the point-wise sharpest bound of the remainder term in the Bahadur representation of the sample quantiles is $(\log \log n / n)^{3 / 4}$. As $\lambda(0)=3 / 4$, we could safely claim the results here could not be further improved for a general class of loss functions $\rho($.$) specified by (A1) and (A2). Nevertheless, it is possible to derive$ stronger results, if the concerned loss functions enjoy a higher degree of smoothness; e.g. (3) in which case $\rho($.$) is the squared loss function. More specifically, suppose that \varphi($.$) is Lipschitz$ continuous and (A1)-(A7) in the Appendix hold with $\lambda_{2}=1 / 2$ and $\lambda_{1}=1$. Then we prove in the Appendix that

$$
\sup _{\underline{x} \in \mathcal{D}}\left|H_{n}\left\{\hat{\beta}_{p}(\underline{x})-\beta_{p}(\underline{x})\right\}-\beta_{n}^{*}(\underline{x})\right|=O\left(\frac{\log n}{n h^{d}}\right) \text { almost surely. }
$$

REMARK 2. The dependence among the observations doesn't have any impact on the rate of the uniform convergence, provided that the degree of the dependence, as measured by the mixing 
coefficient $\gamma[k]$, is weak enough such that (A.3) and (A.4) are satisfied. This is in accordance with the results in Masry (1996), where he proved that for local polynomial estimator of the conditional mean function, the uniform convergence rate is $\left(n h^{d} / \log n\right)^{-1 / 2}$, the same as in the independent case.

REMARK 3. It is of practical interest to provide an explicit rate of decay for the strong mixing coefficient $\gamma[k]$ of the form $\gamma[k]=O\left(1 / k^{c}\right)$ for some $c>0$ (to be determined) for Theorem 3.2 to hold. It is easy to see that, among all the conditions imposed on $\gamma[k]$, the summability condition (A.4) is the most restrictive. We assume that

$$
h=h_{n} \sim(\log n / n)^{\bar{a}} \text { for some } \frac{1}{2(p+s+1)+d} \leq \bar{a}<\frac{1}{d}\left\{1-\frac{4}{\left(1-\lambda_{2}\right) \nu_{2}-4 \lambda_{1}+2\left(1+\lambda_{2}\right)}\right\},
$$

whence (A.2) holds. Algebraic calculations show that (A.4) would be true if

$$
c>\nu_{2} \frac{(1-\bar{a} d)\left\{\left(1-\lambda_{2}\right)(4 N+1)+8 N \lambda_{1}\right\}+10+(4+8 N) \bar{a} d}{2\left(1-\lambda_{2}\right)(1-\bar{a} d) \nu_{2}-8 \bar{a} d+4(1-\bar{a} d)\left(1-\lambda_{2}-2 \lambda_{1}\right)}-1 \equiv c\left(d, p, \nu_{2}, \bar{a}, \lambda_{1}, \lambda_{2}\right) .
$$

Note that we would need the following condition

$$
\nu_{2}>2+\frac{4\left\{\bar{a} d+(1-\bar{a} d) \lambda_{1}\right\}}{(1-\bar{a} d)\left(1-\lambda_{2}\right)}
$$

to secure a positive denominator for (14). As $c\left(d, p, \nu_{2}, \bar{a}, \lambda_{1}, \lambda_{2}\right)$ is decreasing in $\nu_{2}\left(\leq \nu_{1}\right)$, there is a tradeoff between the order $\nu_{1}$ of the moment $E\left|\varphi\left(\varepsilon_{i}\right)\right|^{\nu_{1}}<\infty$ and the decay rate of the strong mixing coefficient $\gamma[k]$ : the existence of higher order moments allows $\gamma[k]$ to decay more slowly.

REMARK 4. It is trivial to generalize the result in Theorem 3.2 to functionals of the Mestimates $\hat{\beta}_{p}(\underline{x})$. Denote the typical elements of $\hat{\beta}_{p}(\underline{x})$ and $\beta_{p}(\underline{x})$ by $\hat{\beta}_{p \underline{r}}(\underline{x})$ and $\beta_{p \underline{r}}(\underline{x}), 0 \leq$ $|\underline{r}| \leq p$ respectively. Suppose $G():. R^{d} \rightarrow R$ satisfies that for any compact set $\mathcal{D} \subset R^{d}$, there exists some constant $C>0$, such that $\left|G^{\prime}\left(\beta_{p \underline{r}}(\underline{x})\right)\right| \leq C$ and $\left|G^{\prime \prime}\left(\beta_{p \underline{r}}(\underline{x})\right)\right| \leq C$ for all $\underline{x} \in \mathcal{D}$. Then with probability 1 ,

$$
\sup _{\underline{x} \in \mathcal{D}}\left|h^{|\underline{r}|}\left\{G\left(\hat{\beta}_{p \underline{r}}(\underline{x})\right)-G\left(\beta_{p \underline{r}}(\underline{x})\right)\right\}-G^{\prime}\left(\beta_{p \underline{r}}(\underline{x})\right) \beta_{n \underline{r}}^{*}(\underline{x})\right|=O\left(\left\{\frac{\log n}{n h^{d}}\right\}^{\lambda(s)}\right) .
$$

The following proposition follows from Theorem 3.2 and the uniform convergence of the sum of weakly dependent zero mean random variables. 
COROLLARY 3.3 Suppose conditions in Theorem 3.2 hold with $s=0$. Then with probability 1 we have, uniformly in $\underline{x} \in \mathcal{D}$,

$H_{n}\left\{\hat{\beta}_{p}(\underline{x})-\beta_{p}(\underline{x})\right\}-E \beta_{n}^{*}(\underline{x})-\frac{W_{p} H_{n}^{-1}}{n h^{d}} S_{n p}^{-1}(\underline{x}) \sum_{i=1}^{n} K_{h}\left(\underline{X}_{i}-\underline{x}\right) \varphi\left(\varepsilon_{i}\right) \mu\left(\underline{X}_{i}-\underline{x}\right)=O\left(\left\{\frac{\log n}{n h^{d}}\right\}^{3 / 4}\right)$.

\section{M-ESTIMATION OF THE ADDITIVE MODEL}

In this section, we apply our main result to derive the properties of a class of estimators in the additive M-regression model (4). In terms of estimating the component functions $m_{k}(),. k=$ $1, \ldots, d$ in (4), the marginal integration method (Linton and Nielsen, 1995) is known to achieve the optimal rate under certain conditions. This involves estimating first the unrestricted Mregression function $m($.$) and then integrating it over some directions. Partition \underline{X}_{i}=\left(x_{1}, \ldots, x_{d}\right)$ as $\underline{X}_{i}=\left(\mathbf{x}_{1 i}, \underline{X}_{2 i}\right)$, where $\mathbf{x}_{1 i}$ is the one dimensional direction of interest and $\underline{X}_{2 i}$ is a $d-1$ dimensional nuisance direction. Let $\underline{x}=\left(x_{1}, \underline{x}_{2}\right)$ and define the functional

$$
\phi_{1}\left(x_{1}\right)=\int m\left(x_{1}, \underline{x}_{2}\right) f_{2}\left(\underline{x}_{2}\right) d \underline{x}_{2}
$$

where $f_{2}\left(\underline{x}_{2}\right)$ is the joint probability density of $\underline{X}_{2 i}$. Under the additive structure $(4), \phi_{1}($.$) is$ $m_{1}($.$) up to a constant. Replace m($.$) in (16)$ with $\hat{\beta}_{0}\left(x_{1}, \underline{x}_{2}\right) \equiv \hat{\beta}_{\underline{0}}(\underline{x})$ given by $(9)$ and $\phi_{1}\left(x_{1}\right)$ can thus be estimated by the sample version of (16):

$$
\phi_{n 1}\left(x_{1}\right)=n^{-1} \sum_{i=1}^{n} \hat{\beta}_{0}\left(x_{1}, \underline{X}_{2 i}\right)
$$

As noted by Linton and Härdle (1996) and Hengartner and Sperlich (2005), cautious choice of the bandwidth is crucial for $\phi_{n 1}($.$) to be asymptotically normal. They suggested different$ bandwidths be used for the direction of interest $X_{1}$ and the $d-1$ dimensional nuisance direction $\underline{X}_{2}$, say $h_{1}$ and $h$ respectively. Sperlich et. al. (1998) provides an extensive study of the small sample properties of the marginal integration estimators, including an evaluation of bandwidth choice.

The following corollary concerns the asymptotic properties of $\phi_{n 1}($.$) .$ 
COROLLARY 4.1 Suppose the support of $\underline{X}$ is $[0,1]^{\otimes d}$ with strictly positive probability density function. Assume that conditions in Proposition 3.3 hold with $\mathrm{T}_{n} \equiv\left\{r(n) / \min \left(h_{1}, h\right)\right\}^{d}$ and the $h^{d}$ replaced by $h_{1} h^{d-1}$ in all the notations defined either in (A.1) or (A.2). If $h_{1} \propto n^{-1 /(2 p+3)}$, $h=O\left(h_{1}\right)$ and (A.2) is modified as

$$
n h_{1} h^{3(d-1)} / \log ^{3} n \rightarrow \infty, n^{-1}\{r(n)\}^{\nu_{2} / 2} d_{n} \log n / M_{n}^{(2)} \rightarrow \infty
$$

then we have

$$
\left(n h_{1}\right)^{1 / 2}\left\{\phi_{n 1}\left(x_{1}\right)-\phi_{1}\left(x_{1}\right)\right\} \stackrel{L}{\rightarrow} N\left(e_{1} W_{p} S_{p}^{-1} B_{1} E \mathbf{m}_{p+1}\left(x_{1}, \underline{X}_{2}\right), \tilde{\sigma}^{2}\left(x_{1}\right)\right),
$$

where $\stackrel{L}{\rightarrow}$ ' stands for convergence in distribution,

$$
\tilde{\sigma}^{2}\left(x_{1}\right)=\left\{\int_{[0,1]^{\otimes d-1}}\left\{f g^{2}\right\}^{-1}\left(x_{1}, \underline{X}_{2}\right) f_{2}^{2}\left(\underline{X}_{2}\right) \sigma^{2}\left(x_{1}, \underline{X}_{2}\right) d \underline{X}_{2}\right\} e_{1} S_{p}^{-1} K_{2} K_{2}^{\top} S_{p}^{-1} e_{1}^{\top},
$$

$\sigma^{2}(\underline{x})=E\left[\varphi^{2}(\varepsilon) \mid \underline{X}=\underline{x}\right]$ and $K_{2}=\int_{[0,1] \otimes d} K(\underline{v}) \mu(\underline{v}) d \underline{v}$. In particular, for the additive quantile regression model, i.e. $\rho(y ; \theta)=(2 q-1)(y-\theta)+|y-\theta|$, we have

$$
\tilde{\sigma}^{2}\left(x_{1}\right)=q(1-q)\left\{\int_{[0,1]^{\otimes d-1}} f^{-1}\left(x_{1}, \underline{X}_{2}\right) f_{\varepsilon}^{-2}\left(0 \mid x_{1}, \underline{X}_{2}\right) f_{2}^{2}\left(\underline{X}_{2}\right) d \underline{X}_{2}\right\} e_{1} S_{p}^{-1} K_{2} K_{2}^{\top} S_{p}^{-1} e_{1}^{\top} .
$$

REMARK 5. For conditions in Corollary 4.1 to hold, we would need $3 d<2 p+5$, i.e. the order $p$ of local polynomial approximation should increase with the dimension of the covariates $\underline{X}$. See also the discussion in Hengartner and Sperlich (2005).

REMARK 6. Besides asymptotic normality, we could also by applying Theorem 3.2 develop Bahadur representations for $\phi_{n 1}\left(x_{1}\right)$, like those assumed in Linton, Sperlich and Van Keilegom (2007). Based on (15), similar results are also applicable to the generalized additive M-regression model, i.e. $G\left(m\left(x_{1}, \ldots, x_{d}\right)\right)=c+m_{1}\left(x_{1}\right)+\ldots+m_{d}\left(x_{d}\right)$ for some known smooth function $G($.$) , in$ which case the marginal integration estimator is defined as the sample average of $G\left(\hat{m}\left(x_{1}, \underline{X}_{2 i}\right)\right)$.

\section{CONCLUSION}

We have obtained an asymptotic expansion for a nonlinear local polynomial M-estimator of a conditional location functional for stationary weakly dependent processes. The approximations 
we have obtained are to a high enough order for many applications based on computing functionals of said estimators. The error from the omitted terms is established in two cases, the smooth case and the unsmooth case, and both cases we achieve what appears to be the optimal rate.

\section{REFERENCES}

Andrews, D.W.K. (1994) Asymptotics for semiparametric econometric models via stochastic equicontinuity. Econometrica 62, 43-72.

Bahadur, R.R. (1966) A note on quantiles in large samples. Annals of Mathematical Statistics $37,577-580$.

Bosq, D. (1998) Nonparametric Statistics for Stochastic Processes. NewYork: Springer-Verlag.

Chen, X., Linton, O. \& I. Van Keilegom (2003) Estimation of Semiparametric Models when the Criterion is not Smooth. Econometrica 71, 1591-1608.

Fan, J., Heckman, N.E. \& Wand, M.P. (1995) Local polynomial kernel regression for generalized linear models and quasi-likelihood functions. Journal of the American Statistical Association 90, 141-150.

Fan, J. \& Gijbels, I. (1996) Local Polynomial Modelling and Its Applications. London: Chapman \& Hall.

Hengartner, N.W. \& Sperlich, S. (2005) Rate optimal estimation with the integration method in the presence of many covariates. Journal of Multivariate Analysis 95, 246 -2 72.

Hall, P. \& Heyde, C.C. (1980) Martingale Limit Theory and Its Application. NewYork: Academic Press.

Hong, S. (2003). Bahadur representation and its application for Local Polynomial Estimates in Nonparametric M-Regression. Journal of Nonparametric Statistics 15, 237-251.

Horowitz, J.L. \& Lee, S. (2005). Nonparametric estimation of an additive quantile regression model. Journal of the American Statistical Association 100, 1238-1249.

Huber, P.J. (1973) Robust regression. Annals of Statistics 1, 799-821. 
Kiefer, J. (1967) On Bahadur's representation of sample quantiles. Annals of Mathematical Statistics. 38, 1323-1342.

Linton, O. (2001) Estimating additive nonparametric models by partial $L_{q}$ Norm: The Curse of Fractionality. Econometric Theory 17, 1037-1350.

Linton, O. \& Nielsen, J.P. (1995) A kernel method of estimating structured nonparametric regression based on marginal integration. Biometrika 82, 93-100.

Linton, O., Sperlich, S., \& I. Van Keilegom (in press). Estimation of a semiparametric transformation model by minimum distance. Annals of Statistics

Linton, O. \& Härdle, W. (1996). Estimation of additive regression models with known links. Biometrika 83, 529-540.

Masry, E. (1996) Multivariate local polynomial regression for time series: uniform strong consistency \& rates. Journal of Time Series Analysis 17, 571-599.

Peng, L. \& Yao, Q. (2003) Least absolute deviation estimation for ARCH \& GARCH models. Biometrika 90, 967-975.

Powell, J.L. (1994) Estimation in semiparametric models. In Engle, R. F. \& McFadden, D. L. (eds.), The Handbook of Econometrics vol. 4, pp. 2444-2521. Amsterdam: North Holland.

Rosenblatt, M. (1956) A central limit theorem and strong mixing conditions. Proceedings of the National Academy of Sciences of the United States of America 4, 43-47.

Sperlich, S., Linton, O. \& Härdle, W. (1998) A Simulation comparison between the backfitting and integration methods of estimating separable nonparametric models. Test 8, 419-458.

Stone, C.J. (1982) Optimal global rates of convergence for nonparametric regression. Annals of Statistics 10, 1040-1053.

Stone, C.J. (1986) The dimensionality reduction principle for generalized additive models. Annals of Statistics 14, 592-606.

Wu, W.B. (2005) On the Bahadur representation of sample quantiles for dependent sequences. Annals of Statistics 33, 1934-1963. 


\section{APPENDIX: Proofs}

We will need the following notations. For any $\lambda_{2} \in(0,1), \lambda_{1} \in\left(\lambda_{2},\left(1+\lambda_{2}\right) / 2\right]$ and $M>2$, define

$$
\begin{aligned}
& d_{n}=\left(n h^{d} / \log n\right)^{-\left(\lambda_{1}+\lambda_{2} / 2\right)}\left(n h^{d} \log n\right)^{1 / 2}, r(n)=\left(n h^{d} / \log n\right)^{\left(1-\lambda_{2}\right) / 2}, \\
& M_{n}^{(1)}=M\left(n h^{d} / \log n\right)^{-\lambda_{1}}, M_{n}^{(2)}=M^{1 / 4}\left(n h^{d} / \log n\right)^{-\lambda_{2}}, \mathrm{~T}_{n}=\{r(n) / h\}^{d},
\end{aligned}
$$

and $\mathrm{L}_{n}$ as the smallest integer such that $\log n(M / 2)^{\mathrm{L}_{n}+1}>n M_{n}^{(2)} / d_{n}$. Let $\|\cdot\|$ denote the Euclidean norm and $C$ be a generic constant, which may take different values in each appearance. Let $\varepsilon_{i} \equiv Y_{i}-m\left(\underline{X}_{i}\right)$ and assume that the following conditions hold.

(A1) For each $y \in \mathcal{R}, \rho(y ; \theta)$ is absolutely continuous in $\theta$, i.e., there exists a function $\varphi(y ; \theta) \equiv$ $\varphi(y-\theta)$ such that for any $\theta \in \mathcal{R}, \rho(y ; \theta)=\rho(y ; 0)+\int_{0}^{\theta} \varphi(y ; t) d t$. The probability density function of $\varepsilon_{i}$ is bounded with $E\left|\varphi\left(\varepsilon_{i}\right)\right|^{\nu_{1}}<\infty$ for some $\nu_{1}>2$, and $E\left\{\varphi\left(\varepsilon_{i}\right) \mid \underline{X}_{i}\right\}=0$ almost surely.

(A2) $\varphi($.$) satisfies the Lipschitz condition in \left(a_{j}, a_{j+1}\right), j=0, \cdots, m$, where $a_{0} \equiv-\infty, a_{m+1} \equiv$ $+\infty$ and $a_{1}<\cdots<a_{m}$ are finite number of jump discontinuity points of $\varphi($.$) .$

(A3) $K($.$) has a compact support, say [-1,1]^{\otimes d}$ and $\left|H_{\underline{j}}(\underline{u})-H_{\underline{j}}(\underline{v})\right| \leq C\|u-v\|$ for all $j$ with $0 \leq|\underline{j}| \leq 2 p+1$, where $H_{\underline{j}}(u)=\underline{u} \underline{j} K(\underline{u})$.

(A4) The probability density function of $\underline{X}, f($.$) is bounded with bounded first order deriva-$ tives. The joint probability density of $\left(\underline{X}_{0}, \underline{X}_{l}\right)$ satisfies $f(\underline{u}, \underline{v} ; l) \leq C<\infty$ for all $l \geq 1$.

(A5) For $\underline{r}$ with $|\underline{r}|=p+1, D^{\underline{r}} m(\underline{x})$ is bounded with bounded first order derivatives.

(A6) The bandwidth $h \rightarrow 0$, such that

$$
n h^{d} / \log n \rightarrow \infty, n h^{d+(p+1) / \lambda_{2}} / \log n<\infty, \quad n^{-1}\{r(n)\}^{\nu_{2} / 2} d_{n} \log n / M_{n}^{(2)} \rightarrow \infty
$$


for some $2<\nu_{2} \leq \nu_{1}$ and the processes $\left\{\left(Y_{i}, \underline{X}_{i}\right)\right\}$ is strongly mixing with mixing coefficient $\gamma[k]$ satisfying

$$
\sum_{k=1}^{\infty} k^{a}\{\gamma[k]\}^{1-2 / \nu_{2}}<\infty \text { for some } a>(p+d+1)\left(1-2 / \nu_{2}\right) / d .
$$

Moreover, the bandwidth $h$ and $\gamma[k]$ should jointly satisfy the following condition

$$
\sum_{n=1}^{\infty} n^{3 / 2} \mathrm{~T}_{n}\left\{\frac{M_{n}^{(1)}}{d_{n}}\right\}^{1 / 2} \frac{\gamma\left[r(n)\left(2^{\nu_{2} / 2} / M\right)^{2 \mathrm{~L}_{n} / \nu_{2}}\right]}{r(n)\left(2^{\nu_{2} / 2} / M\right)^{2 \mathrm{~L}_{n} / \nu_{2}}}\left\{4 M^{2 N}\right\}^{\mathrm{L}_{n}}<\infty, \forall M>0 .
$$

(A7) The conditional density $f_{\underline{X} \mid Y}$ of $\underline{X}$ given $Y$ exists and is bounded. The conditional density function $f_{\left(\underline{X}_{1}, \underline{X}_{l+1}\right) \mid\left(Y_{1}, Y_{l+1}\right)}$ of $\left(\underline{X}_{1}, \underline{X}_{l+1}\right)$ given $\left(Y_{1}, Y_{l+1}\right)$ exists and is bounded for all $l \geq 1$.

REMARK 7. Assumptions on $\varphi($.$) in (A1) and (A2) are satisfied in almost all known robust$ and likelihood type regressions. For example, in $q$ th quantile regression, we have $\varphi(t)=2 q I\{t \geq$ $0\}+(2 q-2) I\{t<0\}$, while for the Huber's function (6), its piecewise derivative is given by

$$
\varphi(t)=t I\{|t|<k\}+\operatorname{sign}(t) k I\{|t| \geq k\} .
$$

Note that the condition $E\left\{\varphi\left(\varepsilon_{i}\right) \mid \underline{X}_{i}\right\}=0$ a.e. is necessary for model specification. Moreover, if the conditional density $f(y \mid \underline{x})$ of $Y$ given $\underline{X}$ is also continuously differentiable with respect to $y$, then as shown in Hong (2003) there exists a constant $C>0$, such that for all small $t$ and $\underline{x}$,

$$
E\left[\{\varphi(Y ; t+a)-\varphi(Y ; a)\}^{2} \mid \underline{X}=\underline{u}\right] \leq C|t|
$$

holds for all $(a, \underline{u})$ in a neighborhood of $(m(\underline{x}), \underline{x})$. Define

$$
G(t, \underline{u})=E\{\varphi(Y ; t) \mid \underline{X}=\underline{u}\}, \quad G_{i}(t, \underline{u})=\left(\partial^{i} / \partial t^{i}\right) G(t, \underline{u}) . i=1,2,
$$

Then it holds that

$$
g(\underline{x})=G_{1}(m(\underline{x}), \underline{x}) \geq C>0, G_{2}(t, \underline{x}) \text { is bounded for all } \underline{x} \in \mathcal{D} \text { and } t \text { near } m(\underline{x}) .
$$

Assumptions (A3)-(A7) are standard for nonparametric smoothing in multivariate time series analysis, see Masry (1996). For example, condition (A.3) is needed to bound the covariance 
of the partial sums of time series as in Lemma 5.5, while (A.4) plays a similar role to (4.7b) in Masry (1996). It guarantees that the dependence of the time series is weakly enough such that the deviance caused by the approximation of dependent random variables by independent ones (through Bradley's strong approximation theorem) is negligible; see Lemma 5.4. Of course, (A.4) is more stringent than (4.7b) in Masry (1996), due to the non-linear nature of the estimates obtained by using the loss function $\rho($.$) instead of the method of least squares.$

Proof of Proposition 3.1. Write $\beta_{n}^{*}(\underline{x})=-W_{p} S_{n, p}^{-1}(\underline{x}) \sum_{i=1}^{n} Z_{n i}(\underline{x}) / n$, where

$$
Z_{n i}(\underline{x})=H_{n}^{-1} h^{-d} K_{h}\left(\underline{X}_{i}-\underline{x}\right) \varphi\left(Y_{i}, \mu\left(\underline{X}_{i}-\underline{x}\right)^{\top} \beta_{p}(\underline{x})\right) \mu\left(\underline{X}_{i}-\underline{x}\right) .
$$

We first focus on $E Z_{n i}(\underline{x})$. Based on (A.6) and (A.7), we have

$$
\begin{aligned}
E\left\{\varphi\left(Y_{i}, \mu\left(\underline{X}_{i}-\underline{x}\right)^{\top} \beta_{p}(\underline{x})\right) \mid \underline{X}_{i}\right\}= & G\left(\mu\left(\underline{X}_{i}-\underline{x}\right)^{\top} \beta_{p}(\underline{x}), \underline{X}_{i}\right) \\
= & -g\left(\underline{X}_{i}\right)\left\{m\left(\underline{X}_{i}\right)-\mu\left(\underline{X}_{i}-\underline{x}\right)^{\top} \beta_{p}(\underline{x})\right\} \\
& +G_{2}\left(\xi_{i}(x), \underline{X}_{i}\right)\left\{m\left(\underline{X}_{i}\right)-\mu\left(\underline{X}_{i}-\underline{x}\right)^{\top} \beta_{p}(\underline{x})\right\}^{2} / 2
\end{aligned}
$$

for some $\xi_{i}(x)$ between $\mu\left(\underline{X}_{i}-\underline{x}\right)^{\top} \beta_{p}(\underline{x})$ and $m\left(\underline{X}_{i}\right)$. Apparently, if $\underline{X}_{i}=\underline{x}+h \underline{v}$, then

$$
m\left(\underline{X}_{i}\right)-\mu\left(\underline{X}_{i}-\underline{x}\right)^{\top} \beta_{p}(\underline{x})=h^{p+1} \sum_{|\underline{k}|=p+1} \frac{D^{\underline{r}} m(\underline{x})}{\underline{k} !} \underline{v}^{\underline{k}}+h^{p+2} \sum_{|\underline{k}|=p+2} \frac{D^{\underline{r}} m(\underline{x})}{\underline{k} !} \underline{v}^{\underline{k}}+o\left(h^{p+2}\right) .
$$

Therefore,

$$
\begin{aligned}
E Z_{n i}(\underline{x})= & h^{p+1} \int K(\underline{v}) f g(\underline{x}+h \underline{v}) \mu(\underline{v}) \sum_{|\underline{k}|=p+1} \frac{D^{\underline{r}} m(\underline{x})}{\underline{k} !} \underline{v}^{\underline{k}} d \underline{v} \\
& +h^{p+2} \int K(\underline{v}) f g(\underline{x}+h \underline{v}) \mu(\underline{v}) \sum_{|\underline{k}|=p+2} \frac{D^{\underline{r}} m(\underline{x})}{\underline{k} !} \underline{v}^{\underline{k}} d \underline{v}+o\left(h^{p+2}\right) \\
\equiv & T_{1}+T_{2} .
\end{aligned}
$$

Now arrange the $N_{p+1}$ elements of the derivatives $D^{\underline{r}} m(\underline{x}) / \underline{r}$ ! for $|\underline{r}|=p+1$ as a column vector $\mathbf{m}_{p+1}(\underline{x})$ using the lexicographical order introduced earlier and define $\mathbf{m}_{p+2}(\underline{x})$ in the similar way. Let the $N \times N_{p+1}$ matrix $B_{n 1}(\underline{x})$ and the $N \times N_{p+2}$ matrix $B_{n 2}(\underline{x})$ be defined as

$$
B_{n 1}(\underline{x})=\left[\begin{array}{c}
S_{n, 0, p+1}(\underline{x}) \\
S_{n, 1, p+1}(\underline{x}) \\
\vdots \\
S_{n, p, p+1}(\underline{x})
\end{array}\right], \quad B_{n 2}(\underline{x})=\left[\begin{array}{c}
S_{n, 0, p+2}(\underline{x}) \\
S_{n, 1, p+2}(\underline{x}) \\
\vdots \\
S_{n, p, p+2}(\underline{x})
\end{array}\right],
$$


where $S_{n, i, p+1}(\underline{x})$ and $S_{n, i, p+2}(\underline{x})$ is as given by (11). Therefore, $T_{1}=h^{p+1} B_{n 1}(\underline{x}) \mathbf{m}_{p+1}(\underline{x})$, $T_{2}=h^{p+2} B_{n 2}(\underline{x}) \mathbf{m}_{p+2}(\underline{x})$, and

$$
E \beta_{n}^{*}(\underline{x})=-W_{p} h^{p+1} S_{n, p}^{-1}(\underline{x}) B_{n 1}(\underline{x}) \mathbf{m}_{p+1}(\underline{x})-W_{p} h^{p+2} S_{n, p}^{-1}(\underline{x}) B_{n 2}(\underline{x}) \mathbf{m}_{p+2}(\underline{x})+o\left(h^{p+2}\right) .
$$

Let $\underline{e}_{i}, i=1, \cdots, d$ be the $d \times 1$ vector having 1 in the $i$ th entry and all other entries 0 . For $0 \leq j \leq p, 0 \leq k \leq p+1$, let $N_{j, k}(\underline{x})$ be a $N_{j} \times N_{k}$ matrix with its $(l, m)$ element given by

$$
\left[N_{j, k}(\underline{x})\right]_{l, m}=\sum_{i=1}^{d} D^{\underline{e}_{i}}\{f g\}(\underline{x}) \int K(\underline{u}) \underline{u}^{\tau_{j}(l)+\tau_{k}(m)+\underline{e}_{i}} d \underline{u},
$$

and use these $N_{j, k}(\underline{x})$ to construct a $N \times N$ matrix $N_{p}(\underline{x})$ and a $N \times N_{p+1}$ matrix $\tilde{M}(\underline{x})$ via

$$
N_{p}(\underline{x})=\left[\begin{array}{cccc}
N_{0,0}(\underline{x}) & N_{0,1}(\underline{x}) & \cdots & N_{0, p}(\underline{x}) \\
N_{1,0}(\underline{x}) & N_{1,1}(\underline{x}) & \cdots & N_{1, p}(\underline{x}) \\
\vdots & \ddots & \vdots & \\
N_{p, 0}(\underline{x}) & N_{p, 1}(\underline{x}) & \cdots & N_{p, p}(\underline{x})
\end{array}\right], \quad \tilde{M}(\underline{x})=\left[\begin{array}{c}
N_{0, p+1}(\underline{x}) \\
N_{1, p+1}(\underline{x}) \\
\vdots \\
N_{p, p+1}(\underline{x})
\end{array}\right] .
$$

Then $S_{n, p}(\underline{x})=\{f g\}(\underline{x}) S_{p}+h N_{p}(\underline{x})+O\left(h^{2}\right), B_{n 1}(\underline{x})=\{f g\}(\underline{x}) B_{1}+h \tilde{M}(\underline{x})+O\left(h^{2}\right)$ and $B_{n 2}(\underline{x})=\{f g\}(\underline{x}) B_{2}+O(h) . \operatorname{As} S_{n, p}^{-1}(\underline{x})=\{f g\}^{-1}(\underline{x}) S_{p}^{-1}-h\{f g\}^{-2}(\underline{x}) S_{p}^{-1} N_{p}(\underline{x}) S_{p}^{-1}+O\left(h^{2}\right)$, we have

$$
\begin{aligned}
-E \beta_{n}^{*}(\underline{x})= & W_{p} h^{p+1}\left[\{f g\}^{-1}(\underline{x}) S_{p}^{-1}-h\{f g\}^{-2}(\underline{x}) S_{p}^{-1} N_{p}(\underline{x}) S_{p}^{-1}\right]\left[\{f g\}(\underline{x}) B_{1}+h \tilde{M}(\underline{x})\right] \mathbf{m}_{p+1}(\underline{x}) \\
& +W_{p} h^{p+2}\{f g\}^{-1}(\underline{x}) S_{p}^{-1}\{f g\}(\underline{x}) B_{2} \mathbf{m}_{p+2}(\underline{x})+o\left(h^{p+2}\right) \\
= & h^{p+1} W_{p} S_{p}^{-1} B_{1} \mathbf{m}_{p+1}(\underline{x})+h^{p+2} W_{p} S_{p}^{-1}\left[\{f g\}^{-1}(\underline{x}) \mathbf{m}_{p+1}(\underline{x})\left\{\tilde{M}(\underline{x})-N_{p}(\underline{x}) S_{p}^{-1} B_{1}\right\}\right. \\
& \left.+B_{2} \mathbf{m}_{p+2}(\underline{x})\right]+o\left(h^{p+2}\right) .
\end{aligned}
$$

We claim that for elements $E \beta_{n \underline{r}}^{*}(\underline{x})$ of $E \beta_{n}^{*}(\underline{x})$ with $p-|\underline{r}|$ even, the $h^{p+1}$ term will vanish. This means for any given $\underline{r}$ with $|\underline{r}| \leq p$ and $\underline{r}_{2}$ with $\left|\underline{r}_{2}\right|=p+1$,

$$
\sum_{0 \leq|\underline{r}| \leq p}\left\{S_{p}^{-1}\right\}_{N\left(\underline{r}_{1}\right), N(\underline{r})} \nu_{\underline{r}}+\underline{r}_{2}=0
$$

To prove this, first note that for any $\underline{r}_{1}$ with $0 \leq\left|\underline{r}_{1}\right| \leq p$ and $\underline{r}_{2}$ with $\left|\underline{r}_{2}\right|=p+1$,

$$
\sum_{0 \leq|\underline{r}| \leq p}\left\{S_{p}^{-1}\right\}_{N\left(\underline{r}_{1}\right), N(\underline{r})} \nu_{\underline{r}+\underline{r}_{2}}=\int \underline{u}^{\underline{r_{2}}} K_{\underline{r}_{1}, p}(\underline{u}) d \underline{u}
$$


where $K_{\underline{r}, p}(\underline{u})=\left\{\left|M_{\underline{r}, p}(\underline{u})\right| /\left|S_{p}\right|\right\} K(\underline{u})$ and $M_{\underline{r}, p}(\underline{u})$ is the same as $S_{p}$, but with the $N(\underline{r})$ column replaced by $\mu(\underline{u})$. Let $c_{i j}$ denote the cofactor of $\left\{S_{p}\right\}_{i, j}$ and expand the determinant of $M_{\underline{r}, p}(\underline{u})$ along the $N(\underline{r})$ column. We can see that

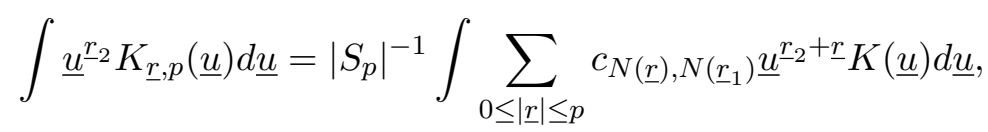

whence (A.9) follows, because $c_{N(\underline{r}), N\left(\underline{r}_{1}\right)} /\left|S_{p}\right|=\left\{S_{p}^{-1}\right\}_{N\left(\underline{r}_{1}\right), N(\underline{r})}$ from the symmetry of $S_{p}$ and a standard result concerning cofactors. As a generalization of Lemma 4 in Fan et. al. (1995) to multivariate case, we can further show that for any $\underline{r}_{1}$ with $0 \leq\left|\underline{r}_{1}\right| \leq p$ and $p-\left|\underline{r}_{1}\right|$ even,

$$
\int \underline{u}^{\underline{r}_{2}} K_{\underline{r}, p}(\underline{u}) d \underline{u}=0, \text { for any }\left|\underline{r}_{2}\right|=p+1
$$

which together with (A.9) leads to (A.8).

We proceed to prove Theorem 3.2. Define $\underline{X}_{i x}=\underline{X}_{i}-\underline{x}, \mu_{i x}=\mu\left(\underline{X}_{i x}\right), K_{i x}=K_{h}\left(\underline{X}_{i x}\right)$ and $\varphi_{n i}(\underline{x} ; t)=\varphi\left(Y_{i} ; \mu_{i x}^{\top} \beta_{p}(\underline{x})+t\right)$. For any $\alpha, \beta \in \mathcal{R}^{N}$, define

$$
\begin{aligned}
\Phi_{n i}(\underline{x} ; \alpha, \beta) & =K_{i x}\left\{\rho\left(Y_{i} ; \mu_{i x}^{\top}\left(\alpha+\beta+\beta_{p}(\underline{x})\right)\right)-\rho\left(Y_{i} ; \mu_{i x}^{\top}\left(\beta+\beta_{p}(\underline{x})\right)\right)-\varphi_{i}(\underline{x} ; 0) \mu_{i x}^{\top} \alpha\right\} \\
& =K_{i x} \int_{\mu_{i x}^{\top} \beta}^{\mu_{i x}^{\top}(\alpha+\beta)}\left\{\varphi_{n i}(\underline{x} ; t)-\varphi_{n i}(\underline{x} ; 0)\right\} d t
\end{aligned}
$$

and $R_{n i}(\underline{x} ; \alpha, \beta)=\Phi_{n i}(\underline{x} ; \alpha, \beta)-E \Phi_{n i}(\underline{x} ; \alpha, \beta)$.

Lemma 5.1 Under assumptions $(A 1)-(A 6)$, we have for all large $M>0$,

$$
\sup _{\substack { x \in \mathcal{D} \\
\begin{subarray}{c}{\alpha \in B_{n}^{(1)} \\
\beta \in B_{n}^{(2)}{ x \in \mathcal { D } \\
\begin{subarray} { c } { \alpha \in B _ { n } ^ { ( 1 ) } \\
\beta \in B _ { n } ^ { ( 2 ) } } }\end{subarray}}\left|\sum_{i=1}^{n} R_{n i}(\underline{x} ; \alpha, \beta)\right| \leq M^{3 / 2} d_{n} \text { almost surely, }
$$

where $B_{n}^{(i)} \equiv\left\{\beta \in \mathcal{R}^{N}:\left|H_{n} \beta\right| \leq M_{n}^{(i)}\right\}, i=1,2$.

Proof. Since $\mathcal{D}$ is compact, it can be covered by a finite number $\mathrm{T}_{n}$ of cubes $\mathcal{D}_{k}=\mathcal{D}_{n, k}$ with 
side length $l_{n}=O\left(\mathrm{~T}_{n}^{-1 / d}\right)=O\left\{h\left(n h^{d} / \log n\right)^{-\left(1-\lambda_{2}\right) / 2}\right\}$ and centers $\underline{x}_{k}=\underline{x}_{n, k}$. Write

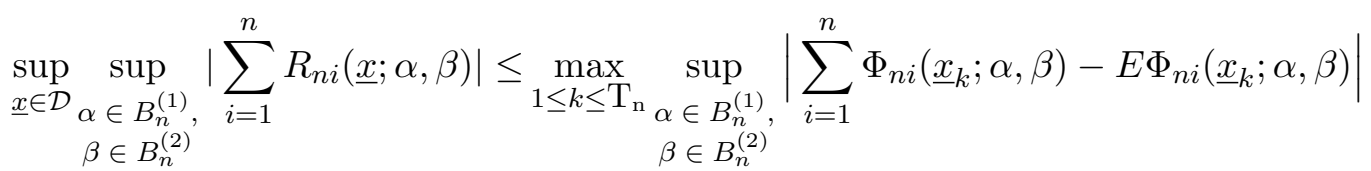

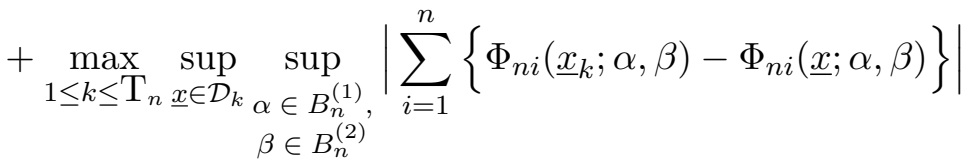

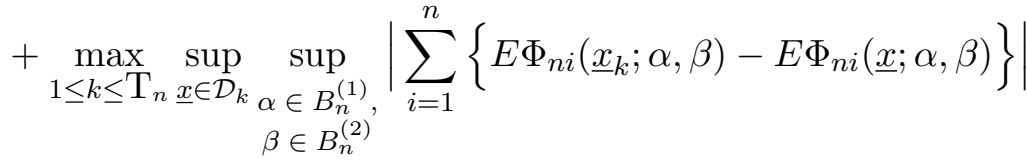

$$
\begin{aligned}
& \equiv Q_{1}+Q_{2}+Q_{3} \text {. }
\end{aligned}
$$

In Lemma 5.2, it is shown that $Q_{2} \leq M^{3 / 2} d_{n} / 3$ almost surely and thus $Q_{3} \leq M^{3 / 2} d_{n} / 3$.

It remains to bound $Q_{1}$. To this end, partition $B_{n}^{(i)}, i=1,2$, into a sequence of disjoint subrectangles $D_{1}^{(i)}, \cdots, D_{J_{1}}^{(i)}$, such that

$$
\left|D_{j_{1}}^{(i)}\right|=\sup \left\{\left|H_{n}(\alpha-\beta)\right|: \alpha, \beta \in D_{j_{1}}^{(i)}\right\} \leq 2 M^{-1} M_{n}^{(i)} / \log n, \quad 1 \leq j_{1} \leq J_{1}
$$

Apparently, $J_{1} \leq(M \log n)^{N}$. For every $1 \leq j_{1} \leq J_{1}, 1 \leq k_{1} \leq J_{1}$, choose a point $\alpha_{j_{1}} \in D_{j_{1}}^{(1)}$ and $\beta_{k_{1}} \in D_{k_{1}}^{(2)}$. Then

$$
\begin{aligned}
Q_{1} \leq & \max _{\substack{1 \leq k \leq \mathrm{T}_{n} \\
1 \leq j_{1}, k_{1} \leq J_{1}}} \sup _{\substack{\alpha \in D_{j_{1}}^{(1)}, D_{k_{1}}^{(2)} \\
\beta \in 1}}\left|\sum_{i=1}^{n}\left\{R_{n i}\left(\underline{x}_{k} ; \alpha_{j_{1}}, \beta_{k_{1}}\right)-R_{n i}\left(\underline{x}_{k} ; \alpha, \beta\right)\right\}\right| \\
& +\max _{\substack{1 \leq k \leq \mathrm{T}_{n} \\
1 \leq j_{1}, k_{1} \leq J_{1}}}\left|\sum_{i=1}^{n} R_{n i}\left(\underline{x}_{k} ; \alpha_{j_{1}}, \beta_{k_{1}}\right)\right|=H_{n 1}+H_{n 2} .
\end{aligned}
$$

We first consider $H_{n 1}$. For each $j_{1}=1, \cdots, J_{1}$ and $i=1,2$, partition each rectangle $D_{j_{1}}^{(i)}$ further into a sequence of subrectangles $D_{j_{1}, 1}^{(i)}, \cdots, D_{j_{1}, J_{2}}^{(i)}$. Repeat this process recursively as follows. Suppose after the $l$ th round, we get a sequence of rectangles $D_{j_{1}, j_{2}, \cdots, j_{l}}^{(i)}$ with $1 \leq j_{k} \leq$ $J_{k}, 1 \leq k \leq l$, then in the $(l+1)$ th round, each rectangle $D_{j_{1}, j_{2}, \cdots, j_{l}}^{(i)}$ is partitioned into a sequence of subrectangles $\left\{D_{j_{1}, j_{2}, \cdots, j_{l}, j_{l+1}}^{(i)}, 1 \leq j_{l} \leq J_{l}\right\}$ such that $\left|D_{j_{1}, j_{2}, \cdots, j_{l}, j_{l+1}}^{(i)}\right|=\sup \left\{\left|H_{n}(\alpha-\beta)\right|: \alpha, \beta \in D_{j_{1}, j_{2}, \cdots, j_{l}, j_{l+1}}^{(i)}\right\} \leq 2 M_{n}^{(i)} /\left(M^{l} \log n\right), 1 \leq j_{l+1} \leq J_{l+1}$, 
where $J_{l+1} \leq M^{N}$. End this process after the $\left(\mathrm{L}_{n}+1\right)$ th round, with $\mathrm{L}_{n}$ given at the beginning of Section 3. Let $D_{l}^{(i)}, i=1,2$, denote the set of all subrectangles of $D_{0}^{(i)}$ after the $l$ th round of partition and a typical element $D_{j_{1}, j_{2}, \cdots, j_{l}}^{(i)}$ of $D_{l}^{(i)}$ is denoted as $D_{\left(j_{l}\right)}^{(i)}$. Choose a point $\alpha_{\left(j_{l}\right)} \in D_{\left(j_{l}\right)}^{(1)}$ and $\beta_{\left(j_{l}\right)} \in D_{\left(j_{l}\right)}^{(2)}$ and define

$$
\begin{aligned}
& V_{l}=\sum_{\substack{\left(j_{l}\right),(k)}} P\left\{\left|\sum_{i=1}^{n}\left\{R_{n i}\left(\underline{x}_{k} ; \alpha_{j_{l}}, \beta_{k_{l}}\right)-R_{n i}\left(\underline{x}_{k} ; \alpha_{j_{l+1}}, \beta_{k_{l+1}}\right)\right\}\right| \geq \frac{M^{3 / 2} d_{n}}{2^{l}}\right\}, 1 \leq l \leq \mathrm{L}_{n}, \\
& Q_{l}=\sum_{\substack{\left(j_{l}\right),\left(k_{l}\right)}} P\left\{\sup _{\substack{\left.\alpha \in D_{\left(j_{l}\right)}^{(1)}, \beta \in D_{\left(k_{l}\right)}^{(2)}\right)}}\left|\sum_{i=1}^{n}\left\{R_{n i}\left(\underline{x}_{k} ; \alpha_{j_{l}}, \beta_{k_{l}}\right)-R_{n i}\left(\underline{x}_{k} ; \alpha, \beta\right)\right\}\right| \geq \frac{M^{3 / 2} d_{n}}{2^{l}}\right\}, 1 \leq l \leq \mathrm{L}_{n}+1 .
\end{aligned}
$$

By (A4), it is easy to see that for any $\alpha \in D_{\left(j_{\mathrm{L}_{n}+1}\right)}^{(1)} \in D_{\mathrm{L}_{n}+1}^{(1)}$ and $\beta \in D_{\left(k_{\mathrm{L}_{n}+1}\right)}^{(2)} \in D_{\mathrm{L}_{n}+1}^{(2)}$,

$$
\left|R_{n i}\left(\underline{x}_{k} ; \alpha, \beta\right)-R_{n i}\left(\underline{x}_{k} ; \alpha_{j_{\mathrm{L}_{n}+1}}, \beta_{k_{\mathrm{L}_{n}+1}}\right)\right| \leq \frac{C M_{n}^{(2)}}{M^{\mathrm{L} n+1} \log n},
$$

which together with the choice of $\mathrm{L}_{n}$ implies that $Q_{\mathrm{L}_{n}+1}=0$. As $Q_{l} \leq V_{l}+Q_{l}, 1 \leq l \leq \mathrm{L}_{n}$,

$$
P\left(H_{n 1}>\frac{M^{3 / 2} d_{n}}{2}\right) \leq \mathrm{T}_{n} Q_{1} \leq \mathrm{T}_{n} \sum_{l=1}^{\mathrm{L}_{n}} V_{l} .
$$

To bound $V_{l}, l=1, \cdots, \mathrm{L}_{n}$, let

$$
W_{n}=\sum_{i=1}^{n} Z_{n i}, Z_{n i} \equiv R_{n i}\left(\underline{x}_{k} ; \alpha_{j_{l}}, \beta_{k_{l}}\right)-R_{n i}\left(\underline{x}_{k} ; \alpha_{j_{l+1}}, \beta_{j_{l+1}}\right) .
$$

Note that by (A2) we have, uniformly in $\underline{x}, \alpha$ and $\beta$, that

$$
\left|\Phi_{n i}(\underline{x} ; \alpha, \beta)\right| \leq C M_{n}^{(1)} .
$$

Therefore, $\left|Z_{n i}\right| \leq C M_{n}^{(1)}$. Using Lemma 5.6, we can apply Lemma 5.4 to each $V_{l}$ with

$$
\begin{aligned}
& B_{1}=C_{1} M_{n}^{(1)}, B_{2}=n h^{d}\left(M_{n}^{(1)}\right)^{2} M_{n}^{(2)}\left\{M^{l} \log n\right\}^{-2 / \nu_{2}}, \\
& r_{n}=r_{n}^{l} \equiv\left(2^{\nu_{2} / 2} / M\right)^{2 l / \nu_{2}} r(n), q=n / r_{n}^{l}, \eta=M^{3 / 2} d_{n} / 2^{l}, \\
& \lambda_{n}=\left(2 C_{1} M_{n}^{(1)} r_{n}^{l}\right)^{-1}, \Psi(n)=C q^{3 / 2} / \eta^{1 / 2} \gamma\left[r_{n}^{l}\right]\left\{r_{n}^{l} M_{n}^{(1)}\right\}^{1 / 2} .
\end{aligned}
$$

Note that $n M_{n}^{(1)} / \eta \rightarrow \infty, r_{n}^{l} \rightarrow \infty$ for all $1 \leq l \leq \mathrm{L}_{n}$ from (A.2) and

$$
\lambda \eta=C M^{1 / 2} \log n M^{2 l / \nu_{2}} / 2^{2 l}, \lambda^{2} B_{2}=C \log n^{1-2 / \nu_{2}} M^{2 l / \nu_{2}} / 2^{2 l}=o(\lambda \eta),
$$


which hold uniformly for all $1 \leq l \leq \mathrm{L}_{n}$. Therefore,

$$
V_{l} \leq\left(\prod_{j=1}^{l+1} J_{j}^{2}\right) 4 \exp \left\{-C_{1} \log n\left(M / 2^{\nu_{2}}\right)^{2 l / \nu_{2}}\right\}+C_{2} \tau_{n}^{l},
$$

where, because $J_{1} \leq 2(M \log n)^{N}$ and $J_{l} \leq 2 M^{N}$ for $2 \leq l \leq L_{n}, \tau_{n}^{l}$ is given by

$$
\tau_{n}^{l}=4^{l} M^{2 N(l+1)}(\log n)^{2 N} n^{3 / 2} \frac{\gamma\left[r_{n}^{l}\right]\left\{M_{n}^{(1)}\right\}^{1 / 2}}{r_{n}^{l}\left\{d_{n}\right\}^{1 / 2}} .
$$

It is tedious but easy to check that for $M$ large enough,

$$
\mathrm{T}_{n} \sum_{l=1}^{\mathrm{L}_{n}}\left[\left(\prod_{j=1}^{l+1} J_{j}^{2}\right) 4 \exp \left\{-C_{1} \log n\left(M / 2^{\nu_{2}}\right)^{2 l / \nu_{2}}\right\}\right] \text { is summable over } n .
$$

As $\gamma\left[r_{n}^{l}\right] / r_{n}^{l}$ is increasing in $l$, we have

$$
\mathrm{T}_{n} \sum_{l=1}^{\mathrm{L}_{n}} \tau_{n}^{l} \leq \mathrm{T}_{n}(\log n)^{2 N} n^{3 / 2} \frac{\left\{M_{n}^{(1)}\right\}^{1 / 2}}{\left\{d_{n}\right\}^{1 / 2}} \frac{\gamma\left[r_{n}^{\mathrm{L}_{n}}\right]}{r_{n}^{\mathrm{L}_{n}}} \prod_{l=1}^{\mathrm{L}_{n}} 4^{l} M^{2 N(l+1)},
$$

which is again summable over $n$ according to (A.4). This along with (A.12) and (A.15) implies that $H_{n 1} \leq M^{3 / 2} d_{n} / 2$ almost surely, using the Borel-Cantelli lemma.

For $H_{n 2}$, first note that

$$
P\left(H_{n 2}>\eta\right) \leq \mathrm{T}_{n} J_{1}^{2} P\left(\left|\sum_{i=1}^{n} R_{n i}\left(\underline{x} ; \alpha_{j_{1}}, \beta_{k_{1}}\right)\right|>\eta\right) .
$$

We apply Lemma 5.4 to quantify $P\left(\mid \sum_{i=1}^{n} R_{n i}\left(\underline{x} ; \alpha_{j_{1}}, \beta_{k_{1}} \mid>\eta\right)\right.$, with $r_{n}=r(n), B_{1}=2 C_{1} M_{n}^{(1)}$, $B_{2}=C_{2} n h^{d}\left(M_{n}^{(1)}\right)^{2} M_{n}^{(2)}, \lambda_{n}=\left\{r(n) M_{n}^{(1)}\right\}^{-1} / 4 C_{1}$ and $\eta=M^{3 / 2} d_{n}$. Then $n B_{1} / \eta \rightarrow \infty$ and

$$
\begin{aligned}
& \lambda_{n} \eta / 4=\left(n h^{d}\right)^{\left(1-\lambda_{2}\right) / 2}(\log n)^{\left(1+\lambda_{2}\right) / 2} /\left\{16 C_{1} r(n)\right\}=M^{1 / 2} \log n /\left(16 C_{1}\right), \\
& \lambda_{n}^{2} B_{2}=M^{1 / 4}\left(n h^{d}\right)^{1-\lambda_{2}}(\log n)^{\lambda_{2}} /\left\{16 C_{1}^{2} r^{2}(n)\right\}=M^{1 / 4} \log n /\left(16 C_{1}^{2}\right), \\
& \Psi(n) \equiv q_{n}\left\{n B_{1} / \eta\right\}^{1 / 2} \gamma\left[r_{n}\right]=\mathrm{T}_{n} J_{1}^{2} q(n)^{3 / 2} / \eta^{1 / 2} \gamma[r(n)]\left\{r(n) M_{n}^{(1)}\right\}^{1 / 2},
\end{aligned}
$$

where $\Psi(n)$ is summable over $n$ under condition (A.4). Therefore,

$$
P\left(H_{n 2}>\eta\right) \leq 2 \mathrm{~T}_{n} J_{1}^{2} / n^{b}+\Psi(n), b=\frac{1}{16 C_{1}}\left(M^{1 / 2}-M^{1 / 4} C_{2} / C_{1}\right) .
$$

By selecting $M$ large enough, we can ensure that the right hand side of (A.17) is summable over $n$. Thus, for $M$ large enough, $H_{n 2} \leq M^{3 / 2} d_{n}$ almost surely. By (A.39), we know for large $M$, $Q_{1} \leq M^{3 / 2} d_{n}$ almost surely.

The quantification of $Q_{2}$ is relatively more involved, so we put it as a separate lemma. 
Lemma 5.2 Under conditions in Lemma 5.1, $Q_{2} \leq M^{3 / 2} d_{n} / 3$ almost surely.

Proof. Let $\underline{X}_{i k}=\underline{X}_{i}-\underline{x}_{k}, \mu_{i k}=\mu\left(\underline{X}_{i k}\right)$ and $K_{i k}=K_{h}\left(\underline{X}_{i k}\right)$. Write $\Phi_{n i}\left(\underline{x}_{k} ; \alpha, \beta\right)-\Phi_{n i}(x ; \alpha, \beta)=$ $\xi_{i 1}+\xi_{i 2}+\xi_{i 3}$, where

$$
\begin{aligned}
& \xi_{i 1}=\left(K_{i k} \mu_{i k}-K_{i x} \mu_{i x}\right)^{\top} \alpha \int_{0}^{1}\left\{\varphi_{n i}\left(\underline{x}_{k} ; \mu_{i k}^{\top}(\beta+\alpha t)\right)-\varphi_{n i}\left(\underline{x}_{k} ; 0\right)\right\} d t \\
& \xi_{i 2}=K_{i x} \mu_{i x}^{\top} \alpha \int_{0}^{1}\left\{\varphi_{n i}\left(\underline{x}_{k} ; \mu_{i k}^{\top}(\beta+\alpha t)\right)-\varphi_{n i}\left(x ; \mu_{i x}^{\top}(\beta+\alpha t)\right)\right\} d t \\
& \xi_{i 3}=K_{i x} \mu_{i x}^{\top} \alpha\left\{\varphi_{n i}(x ; 0)-\varphi_{n i}\left(\underline{x}_{k} ; 0\right)\right\} .
\end{aligned}
$$

Then $P\left(Q_{2}>M^{3 / 2} d_{n} / 3\right) \leq \mathrm{T}_{n}\left(P_{n 1}+P_{n 2}+P_{n 3}\right)$, with

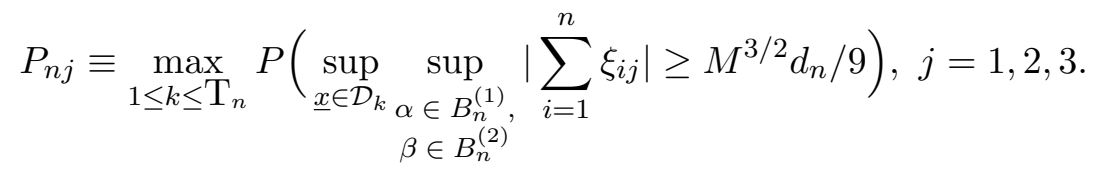

Based on Borel-Cantelli lemma, $Q_{2} \leq M^{3 / 2} d_{n}$ almost surely, if $\sum_{n} \mathrm{~T}_{n} P_{n j}<\infty, j=1,2,3$.

We first study $P_{n 1}$. For any fixed $\alpha \in B_{n}^{(1)}$ and $\beta \in B_{n}^{(2)}$, let $I_{i k}^{\alpha, \beta}=1$, if there exists some $t \in[0,1]$, such that there are discontinuity points of $\varphi\left(Y_{i} ; \theta\right)$ between $\left.\mu_{i k}^{\top}\left(\beta_{p}\left(\underline{x}_{k}\right)+\beta+\alpha t\right)\right)$ and $\mu_{i k}^{\top} \beta_{p}\left(\underline{x}_{k}\right)$; and $I_{i k}^{\alpha, \beta}=0$, otherwise. Write $\xi_{i 1}=\xi_{i 1} I_{i k}^{\alpha, \beta}+\xi_{i 1}\left(1-I_{i k}^{\alpha, \beta}\right)$. Note that by (A3), $\left|\left(K_{i k} \mu_{i k}-K_{i x} \mu_{i x}\right)^{\top} \alpha\right| \leq C_{2} M_{n}^{(1)} l_{n} / h$. Then by (A2) and the fact that $\left|\mu_{i k}^{\top}(\beta+\alpha t)\right| \leq C M_{n}^{(2)}$, we have $\left|\xi_{i 1}\left(1-I_{i k}^{\alpha, \beta}\right)\right| \leq C M_{n}^{(2)} M_{n}^{(1)} l_{n} / h$ uniformly in $i, \alpha, \beta$ and $\underline{x} \in \mathcal{D}_{k}$. Define $U_{i k}=I\left\{\left|\underline{X}_{i k}\right| \leq\right.$ $2 h\}$, whence $\xi_{i 1}=\xi_{i 1} U_{i k}$ since $l_{n}=o(h)$. Therefore,

$$
\begin{aligned}
P\left(\sup _{\substack{\alpha \in B_{n}^{(1)}, \underline{x} \in \mathcal{D}_{k} \\
\beta \in B_{n}^{(2)}}} \sup _{i=1}\left|\sum_{i 1}\left(1-I_{i k}^{\alpha, \beta}\right)\right|>\frac{M^{3 / 2} d_{n}}{18}\right) & \leq P\left(\sum_{i=1}^{n} U_{i k}>\frac{M^{1 / 4} n h^{d}}{18 C}\right) \\
& \leq P\left(\left|\sum_{i=1}^{n} U_{i k}-E U_{i k}\right|>\frac{M^{1 / 4} n h^{d}}{36 C}\right)
\end{aligned}
$$

where the second inequality follows from the fact that $\operatorname{Var}\left(\sum_{i=1}^{n} I\left\{\left|\underline{X}_{i k}\right| \leq 2 h\right)=O\left(n h^{d}\right)\right.$ implied by Lemma 5.5. To quantify (A.18), we apply Lemma 5.4 with $B_{1}=1, \eta=M^{1 / 4} n h^{d} /(18 C), B_{2}=$ $n h^{d}, r_{n}=r(n)$. As $\lambda_{n} \eta=C M^{1 / 4} \log n\left(n h^{d} / \log n\right)^{\left(1+\lambda_{2}\right) / 2}, \lambda_{n}^{2} B_{2}=o\left(\lambda_{n} \eta\right)$ and $\mathrm{T}_{n} \Psi_{n}$ is summable over $n$ under condition (A.4), we know that

$$
\mathrm{T}_{n} P\left(\sup _{\substack{\alpha \in B_{n}^{(1)} \\ \beta \in B_{n}^{(2)}}}\left|\sum_{i=1}^{n} \xi_{i 1}\left(1-I_{i k}^{\alpha, \beta}\right)\right|>M^{3 / 2} d_{n} / 18\right) \text { is summable over } n,
$$


whence $\sum_{n} \mathrm{~T}_{n} P_{n 1}<\infty$, is equivalent to

$$
\mathrm{T}_{n} P\left(\sup _{\substack{\alpha \in B_{n}^{(1)}, \beta \in B_{n}^{(2)}}}\left|\sum_{i=1}^{n} \xi_{i 1} I_{i k}^{\alpha, \beta}\right|>M^{3 / 2} d_{n} / 18\right) \text { is summable over } n .
$$

To prove (A.20), first note that $I_{i k}^{\alpha, \beta} \leq I\left\{\varepsilon_{i} \in S_{i ; k}^{\alpha, \beta}\right\}$, where

$$
\begin{aligned}
S_{i ; k}^{\alpha, \beta} & =\bigcup_{j=1}^{m} \bigcup_{t \in[0,1]}\left[a_{j}-A\left(\underline{X}_{i}, \underline{x}_{k}\right)+\mu_{i k}^{\top}(\beta+\alpha t), a_{j}-A\left(\underline{X}_{i}, \underline{x}_{k}\right)\right] \\
& \subseteq \bigcup_{j=1}^{m}\left[a_{j}-C M_{n}^{(2)}, a_{j}+C M_{n}^{(2)}\right] \equiv D_{n}, \quad \text { for some } C>0 \\
A\left(\underline{x}_{1}, \underline{x}_{2}\right) & =(p+1) \sum_{|\underline{r}|=p+1} \frac{1}{\underline{r} !}\left(\underline{x}_{1}-\underline{x}_{2}\right)^{\underline{r}} \int_{0}^{1} D^{-\underline{r}} m\left(\underline{x}_{2}+w\left(\underline{x}_{1}-\underline{x}_{2}\right)\right)(1-w)^{p} d w
\end{aligned}
$$

where in the derivation of $S_{i ; k}^{\alpha, \beta} \subseteq D_{n}$, we have used the fact that $\left|\underline{X}_{i k}\right| \leq 2 h$ and $A\left(\underline{X}_{i}, \underline{x}_{k}\right)=$ $O\left(h^{p+1}\right)=O\left(M_{n}^{(2)}\right)$ uniformly in $i$. As $I_{i k}^{\alpha, \beta} \leq I\left\{\varepsilon_{i} \in D_{n}\right\}$, we have $\left|\xi_{i 1}\right| I_{i k}^{\alpha, \beta} \leq\left|\xi_{i 1}\right| U_{n i}$, where $U_{n i} \equiv I\left(\left|\underline{X}_{i k}\right| \leq 2 h\right) I\left\{\varepsilon_{i} \in D_{n}\right\}$, which is independent of the choice of $\alpha$ and $\beta$. Therefore,

$$
\begin{aligned}
P\left(\sup _{\substack{\alpha \in B_{n}^{(1)} \\
\beta \in B_{n}^{(2)}}}\left|\sum_{i=1}^{n} \xi_{i 1} I_{i k}^{\alpha, \beta}\right|>M^{3 / 2} d_{n} / 18\right) & \leq P\left(\sum_{i=1}^{n} U_{n i}>M^{1 / 2} n h^{d} M_{n}^{(2)} /(18 C)\right) \\
& \leq P\left(\sum_{i=1}^{n}\left(U_{n i}-E U_{n i}\right)>\frac{M^{1 / 2} n h^{d} M_{n}^{(2)}}{36 C}\right)
\end{aligned}
$$

where the first inequality is because $\left|\xi_{i 1}\right| \leq C M_{n}^{(1)} l_{n} / h$ and the second one is because $E U_{n i}=$ $O\left(h^{d} M_{n}^{(2)}\right)$ by (A1). As $E U_{n i}^{2}=E U_{n i}$, by Lemma 5.5, we know that $\operatorname{Var}\left(\sum_{i=1}^{n} U_{n i}\right)=C n h^{d} M_{n}^{(2)}$. We can then apply Lemma 5.4 to the last term in (A.21) with

$$
B_{2}=C n h^{d} M_{n}^{(2)}, B_{1} \equiv 1, r_{n}=r(n), \eta \equiv M^{1 / 2} n h^{d} M_{n}^{(2)} /(36 C) .
$$

Apparently, $\lambda_{n} \eta=C \log n\left(n h^{d} / \log n\right)^{\left(1-\lambda_{2}\right) / 2}$ and $\lambda_{n}^{2} B_{2}=o\left(\lambda_{n} \eta\right)$. As in this case $T_{n} \Psi_{n}$ is still summable over $n$ by (A.4), (A.20) thus follows.

For $P_{n 2}$, first note that using approach for $P_{n 1}$, we can show that

$$
\mathrm{T}_{n} P\left(\sup _{\substack{\alpha \in B_{n}^{(1)}, \underline{x} \in \mathcal{D}_{k} \\ \beta \in B_{n}^{(2)}}}\left|\sum_{i=1}^{n}\left\{\xi_{i 2}-\tilde{\xi}_{i 2}\right\}\right| \geq M^{3 / 2} d_{n} / 18\right) \text { is summable over } n .
$$


where

$$
\tilde{\xi}_{i 2}=K_{i k} \mu_{i k}^{\top} \alpha \int_{0}^{1}\left\{\varphi_{n i}\left(\underline{x}_{k} ; \mu_{i k}^{\top}(\beta+\alpha t)\right)-\varphi_{n i}\left(x ; \mu_{i x}^{\top}(\beta+\alpha t)\right)\right\} d t .
$$

Therefore, we would have $\sum \mathrm{T}_{n} P_{n 2}<\infty$, if

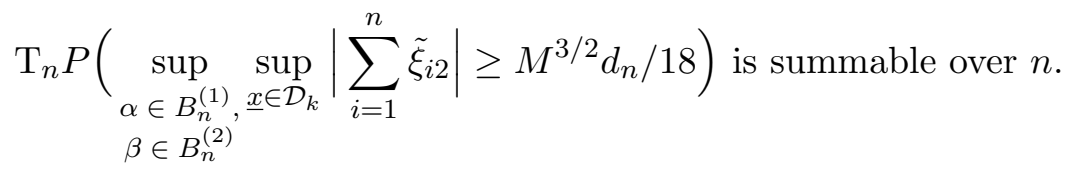

For any fixed $\alpha \in B_{n}^{(1)}, \beta \in B_{n}^{(2)}$ and $\underline{x} \in \mathcal{D}_{k}$, let $I_{i ; k, x}^{\alpha, \beta}=1$, if there exists some interval $\left[t_{1}, t_{2}\right] \subseteq[0,1]$, such that

$$
Y_{i}-\mu_{i k}^{\top}\left(\beta_{p}\left(\underline{x}_{k}\right)+\beta+\alpha t\right) \leq a_{j} \leq Y_{i}-\mu_{i x}^{\top}\left(\beta_{p}(\underline{x})+\beta+\alpha t\right), \forall t \in\left[t_{1}, t_{2}\right]
$$

with $a_{j} \in\left\{a_{1}, \cdots, a_{m}\right\}$; and $I_{i ; k, x}^{\alpha, \beta}=0$, otherwise. Write $\tilde{\xi}_{i 2}=\tilde{\xi}_{i 2} I_{i ; k, x}^{\alpha, \beta}+\tilde{\xi}_{i 2}\left(1-I_{i ; k, x}^{\alpha, \beta}\right)$. Note that $K_{i k} \mu_{i k}^{\top} \alpha=O\left(M_{n}^{(1)}\right)$ and $\varphi_{n i}\left(\underline{x}_{k} ; \mu_{i k}^{\top}(\beta+\alpha t)\right)-\varphi_{n i}\left(x ; \mu_{i x}^{\top}(\beta+\alpha t)\right)=O\left(M_{n}^{(2)} l_{n} / h\right)$ if $I_{i ; k, x}^{\alpha, \beta}=0$. Then again as $\tilde{\xi}_{i 2}=\tilde{\xi}_{i 2} I\left\{\left|\underline{X}_{i k}\right| \leq 2 h\right\}$, we have similar to (A.19) that

$$
\mathrm{T}_{n} P\left(\sup _{\substack{\alpha \in B_{n}^{(1)} \\ \beta \in B_{n}^{(2)}}}\left|\sum_{i=1}^{n} \tilde{\xi}_{i 2}\left(1-I_{i ; k, x}^{\alpha, \beta}\right)\right|>M^{3 / 2} d_{n} / 18\right) \text { is summable over } n .
$$

Therefore, by (A.22), to show $\sum \mathrm{T}_{n} P_{n 2}<\infty$, it is sufficient to show that

$$
\mathrm{T}_{n} P\left(\sup _{\substack{\alpha \in B_{n}^{(1)} \\ \beta \in B_{n}^{(2)}}} \sup _{\substack{x \in \mathcal{D}_{k} \\ \beta}}\left|\sum_{i=1}^{n} \tilde{\xi}_{i 2} I_{i ; k, x}^{\alpha, \beta}\right| \geq M^{3 / 2} d_{n} / 36\right) \text { is summable over } n .
$$

To this end, define $\epsilon_{i}=\varepsilon_{i}+A\left(\underline{X}_{i}, \underline{x}_{k}\right)$. Then $I_{i ; k, x}^{\alpha, \beta}=1$, i.e. (A.23) is equivalent to

$$
A\left(\underline{X}_{i}, \underline{x}_{k}\right)-A\left(\underline{X}_{i}, \underline{x}\right)+\mu_{i x}^{\top}(\beta+\alpha t) \leq \epsilon_{i}-a_{j} \leq \mu_{i k}^{\top}(\beta+\alpha t), \forall t \in\left[t_{1}, t_{2}\right] .
$$

Let $\delta_{n} \equiv M_{n}^{(2)} l_{n} / h$. Then $\left|A\left(\underline{X}_{i}, \underline{x}_{k}\right)-A\left(\underline{X}_{i}, \underline{x}\right)\right| \leq C \delta_{n},\left|\left(\mu_{i k}-\mu_{i x}\right)^{\top} \beta\right| \leq C \delta_{n}$ and (A.25) thus implies that

$$
-2 C \delta_{n}+\mu_{i k}^{\top}(\beta+\alpha t) \leq \epsilon_{i}-a_{j} \leq \mu_{i k}^{\top}(\beta+\alpha t)+2 C \delta_{n}, \quad \forall t \in\left[t_{1}, t_{2}\right]
$$

Without loss of generality, assume $\mu_{i k}^{\top} \alpha>0$. Then from (A.26) we can see that

$$
-2 C \delta_{n}+\mu_{i k}^{\top}\left(\beta+\alpha t_{2}\right) \leq \epsilon_{i}-a_{j} \leq \mu_{i k}^{\top}\left(\beta+\alpha t_{1}\right)+2 C \delta_{n}
$$


which in turn means that if $I_{i ; k, x}^{\alpha, \beta}=1$, then $\left|\xi_{i 2}\right| \leq C\left(t_{2}-t_{1}\right)\left|\mu_{i k}^{\top} \alpha\right| \leq 4 C \delta_{n}$ uniformly in $i, \alpha \in B_{n}^{(1)}, \beta \in B_{n}^{(2)}$ and $\underline{x} \in \mathcal{D}_{k}$. Therefore, as $\tilde{\xi}_{i 2}=\tilde{\xi}_{i 2} I\left\{\left|\underline{X}_{i k}\right| \leq 2 h\right\}$, we have

$$
\begin{aligned}
& P\left(\sup _{\substack{\alpha \in B_{n}^{(1)} \\
\beta \in B_{n}^{(2)}}} \sup _{x \in \mathcal{D}_{k}}\left|\sum_{i=1}^{n} \tilde{\xi}_{i 2} I_{i ; k, x}^{\alpha, \beta}\right| \geq \frac{M^{3 / 2} d_{n}}{36}\right) \\
& \leq P\left(\sup _{\substack{\alpha \in B_{n}^{(1)} \\
\beta \in B_{n}^{(2)}}} \sup _{\substack{x \in \mathcal{D}_{k} \\
k \in i=1}} \sum_{i=1}^{n} I\left\{\left|\underline{X}_{i k}\right| \leq 2 h\right\} I_{i ; k, x}^{\alpha, \beta} \geq \frac{M^{5 / 4} n h^{d} M_{n}^{(1)}}{36 C}\right) .
\end{aligned}
$$

We will bound $I_{i ; k, x}^{\alpha, \beta}$ by a random variable that is independent of the choice of $\alpha \in B_{n}^{(1)}$ and $\underline{x} \in D_{k}$. By the definition of $I_{i ; k, x}^{\alpha, \beta}$ and (A.27), the necessary condition for $I_{i ; k, x}^{\alpha, \beta}=1$ is

$$
\epsilon_{i} \in \bigcup_{j=1}^{m}\left[a_{j}+\mu_{i k}^{\top} \beta-2 M_{n}^{(1)}, a_{j}+\mu_{i k}^{\top} \beta+2 M_{n}^{(1)}\right] \equiv D_{n i}^{\beta},
$$

which is indeed independent of the choice of $\alpha$ and $\underline{x} \in \mathcal{D}_{k}$. Therefore,

$$
\begin{aligned}
& P\left(\sup _{\substack{\alpha \in B_{n}^{(1)}, \underline{x} \in \mathcal{D}_{k} \\
\beta \in B_{n}^{(2)}}} \sum_{i=1}^{n} I\left\{\left|\underline{X}_{i k}\right| \leq 2 h\right\} I_{i ; k, x}^{\alpha, \beta} \geq \frac{M^{5 / 4} n h^{d} M_{n}^{(1)}}{36 C}\right) \\
\leq & P\left(\sup _{\beta \in B_{n}^{(2)}} \sum_{i=1}^{n} I\left\{\left|\underline{X}_{i k}\right| \leq 2 h\right\} I\left\{\epsilon_{i} \in D_{n i}^{\beta}\right\} \geq \frac{M^{5 / 4} n h^{d} M_{n}^{(1)}}{36 C}\right) .
\end{aligned}
$$

Now we partition $B_{n}^{(2)}$ into a sequence of subrectangles $S_{1}, \cdots, S_{m}$, such that

$$
\left|S_{l}\right|=\sup \left\{\left|H_{n}\left(\beta-\beta^{\prime}\right)\right|: \beta, \beta^{\prime} \in S_{l}\right\} \leq M_{n}^{(1)}, \quad 1 \leq l \leq m
$$

Obviously, $m \leq\left(M_{n}^{(2)} / M_{n}^{(1)}\right)^{N}=M^{-3 N / 4}\left(n h^{d} / \log n\right)^{\left(\lambda_{1}-\lambda_{2}\right) N}$. Choose a point $\beta_{l} \in S_{l}$ for each $1 \leq l \leq m$, and thus

$$
\begin{aligned}
& P\left(\sup _{\beta \in B_{n}^{(2)}} \sum_{i=1}^{n} I\left\{\left|\underline{X}_{i k}\right| \leq 2 h\right\} I\left\{\epsilon_{i} \in D_{n i}^{\beta}\right\} \geq \frac{M^{5 / 4} n h^{d} M_{n}^{(1)}}{36 C}\right) \\
\leq & m P\left(\sum_{i=1}^{n} I\left\{\left|\underline{X}_{i k}\right| \leq 2 h\right\} I\left\{\epsilon_{i} \in D_{n i}^{\beta_{l}}\right\} \geq \frac{M^{5 / 4} n h^{d} M_{n}^{(1)}}{72 C}\right) \\
& +m P\left(\sup _{\beta^{\prime} \in S_{l}} \sum_{i=1}^{n} I\left\{\left|\underline{X}_{i k}\right| \leq 2 h\right\}\left|I\left\{\epsilon_{i} \in D_{n i}^{\beta_{l}}\right\}-I\left\{\epsilon_{i} \in D_{n i}^{\beta^{\prime}}\right\}\right| \geq \frac{M^{5 / 4} n h^{d} M_{n}^{(1)}}{72 C}\right) \\
\equiv & m\left(T_{1}+T_{2}\right) .
\end{aligned}
$$


We deal with $T_{1}$ first. Let

$$
U_{n i}^{j} \equiv I\left\{\left|\underline{X}_{i k}\right| \leq 2 h\right\} I\left\{\epsilon_{i} \in D_{n i}^{\beta_{l}}\right\}
$$

Then by the definition of $D_{n i}^{\beta_{j}}$ given in (A.29), $E U_{n i}^{j}=O\left(h^{d} M_{n}^{(1)}\right)<M^{5 / 4} h^{d} M_{n}^{(1)} /(144 C)$ for large $M$ and we have

$$
T_{1} \leq P\left(\sum_{i=1}^{n}\left(U_{n i}^{j}-E U_{n i}^{j}\right) \geq \frac{M^{5 / 4} n h^{d} M_{n}^{(1)}}{144 C}\right) .
$$

We can thus apply Lemma 5.4 to the quantity on the right hand side with $B_{1} \equiv 1, B_{2}$ given by (A.51), $r_{n}=r(n)$ and $\eta \propto M^{5 / 4} n h^{d} M_{n}^{(1)}$, and $\lambda_{n}=1 /\left(2 r_{n}\right)$. It follows that

$$
\lambda_{n} \eta=C M^{5 / 4} \log n\left(n h^{d} / \log n\right)^{\left(1+\lambda_{2}\right) / 2-\lambda_{1}}, \lambda_{n}^{2} B_{2}=C \log n\left(n h^{d} / \log n\right)^{-2\left(\lambda_{1}-\lambda_{2}\right) / \nu_{2}} .
$$

As $\left(1+\lambda_{2}\right) / 2 \geq \lambda_{1}$ and $\lambda_{2}<\lambda_{1}$, we have $T_{1}=O\left(n^{-b}\right)$ for any $b>0$.

For $T_{2}$, note that as $\left|\mu_{i k}^{\top}\left(\beta-\beta_{l}\right)\right| \leq C M_{n}^{(1)}$ for any $\beta \in S_{l}, 1 \leq l \leq m$, we have

$$
\begin{aligned}
\left|I\left\{\epsilon_{i} \in D_{n i}^{\beta_{l}}\right\}-I\left\{\epsilon_{i} \in D_{n i}^{\beta}\right\}\right| & =I\left\{\epsilon_{i} \in D_{n i}^{\beta_{l}} \backslash D_{n i}^{\beta}\right\} \\
& \leq I\left\{\epsilon_{i} \in \bigcup_{j=1}^{m}\left[a_{j}+\mu_{i k}^{\top} \beta_{l}-C M_{n}^{(1)}, a_{j}+\mu_{i k}^{\top} \beta_{l}+C M_{n}^{(1)}\right]\right\} \equiv U_{n i},
\end{aligned}
$$

for some $C>0$, which is independent of the choice of $\beta \in S_{l}$. Therefore,

$$
T_{2} \leq P\left(\sum_{i=1}^{n} I\left\{\left|\underline{X}_{i k}\right| \leq 2 h\right\} U_{n i} \geq \frac{M^{5 / 4} n h^{d} M_{n}^{(1)}}{72 C}\right),
$$

which can be dealt with similarly as with $T_{1}$ and thus $T_{2}=O\left(n^{-b}\right)$ for any $b>0$. Thus from (A.28), (A.30) and (A.31), we can claim that (A.24) is true and thus $\mathrm{T}_{n} P_{n 2}$ is summable over $n$.

Dealing with $P_{n 3}$ is simpler, as no $\beta$ is involved in $\xi_{i 3}$. For any given $\underline{x} \in \mathcal{D}_{k}$, let $I_{i ; k, x}=1$, if there is a discontinuity point of $\varphi\left(Y_{i} ; \theta\right)$ between $\mu_{i k}^{\top} \beta_{p}\left(\underline{x}_{k}\right)$ and $\mu_{i x}^{\top} \beta_{p}(\underline{x})$; and $I_{i ; k, x}=0$, otherwise. Write $\xi_{i 3}=\xi_{i 3} I_{i ; k, x}+\xi_{i 3}\left(1-I_{i ; k, x}\right)$. Again by (A2) and the fact that $\left|K_{i x} \mu_{i x}^{\top} \alpha\right|=$ $O\left(M_{n}^{(1)}\right)$ and $\left|\mu_{i k}^{\top} \beta_{p}\left(\underline{x}_{k}\right)-\mu_{i x}^{\top} \beta_{p}(\underline{x})\right|=\left|A\left(\underline{X}_{i}, \underline{x}_{k}\right)-A\left(\underline{X}_{i}, \underline{x}\right)\right|=O\left(M_{n}^{(2)} l_{n} / h\right)$, we have similar to (A.19) that

$$
\mathrm{T}_{n} P\left(\sup _{\substack{\alpha \in B_{n}^{(1)} \\ \underline{x} \in \mathcal{D}_{k}}}\left|\sum_{i=1}^{n} \xi_{i 3}\left(1-I_{i ; k, x}\right)\right|>M^{3 / 2} d_{n} / 18\right) \text { is summable over } n .
$$


It's easy to see that $I_{i ; k, x} \leq I\left\{\varepsilon_{i}+A\left(\underline{X}_{i}, \underline{x}_{k}\right) \in S_{i ; k, x}\right\}$, where

$$
\begin{aligned}
S_{i ; k, x} & =\bigcup_{j=1}^{m} \bigcup_{t \in[0,1]}\left[a_{j}-\left|A\left(\underline{X}_{i}, \underline{x}_{k}\right)-A\left(\underline{X}_{i}, \underline{x}\right)\right|, a_{j}+\left|A\left(\underline{X}_{i}, \underline{x}_{k}\right)-A\left(\underline{X}_{i}, \underline{x}\right)\right|\right] \\
& \subseteq \bigcup_{j=1}^{m}\left[a_{j}-C M_{n}^{(2)} l_{n} / h, a_{j}+C M_{n}^{(2)} l_{n} / h\right] \equiv D_{n}, \text { for some } C>0 .
\end{aligned}
$$

Therefore, $\left|\xi_{i 3}\right| I_{i ; k, x}=\left|\xi_{i 3}\right| I\left\{\left|\underline{X}_{i k}\right| \leq 2 h\right\} I_{i ; k, x} \leq U_{n i}$, with

$$
U_{n i} \equiv M_{n}^{(1)} I\left\{\left|\underline{X}_{i k}\right| \leq 2 h\right\} I\left\{\varepsilon_{i}+A\left(\underline{X}_{i}, \underline{x}_{k}\right) \in D_{n}\right\}
$$

which is independent of the choice of $\alpha \in B_{n}^{(1)}$ and $\underline{x} \in \mathcal{D}_{k}$. Therefore,

$$
\mathrm{T}_{n} P\left(\sup _{\substack{\alpha \in B_{n}^{(1)} \\ \underline{x} \in \mathcal{D}_{k}}}\left|\sum_{i=1}^{n} \xi_{i 3} I_{i ; k, x}\right|>M^{3 / 2} d_{n} / 18\right) \leq \mathrm{T}_{n} P\left(\sum_{i=1}^{n}\left[U_{n i}-E U_{n i}\right]>M^{3 / 2} d_{n} / 36\right),
$$

where we have used the fact that $E U_{n i}=O\left(h^{d} M_{n}^{(1)} M_{n}^{(2)} l_{n} / h\right)=O\left(d_{n} / n\right)$. We will have $\sum \mathrm{T}_{n} P_{n 3}<\infty$ if the right hand side in (A.33) is summable over $n$, i.e.

$$
\mathrm{T}_{n} P\left(\sum_{i=1}^{n}\left[U_{n i}-E U_{n i}\right]>M^{3 / 2} d_{n} / 36\right) \text { is summable over } n .
$$

It's easy to check that Lemma 5.5 again holds with $\psi_{\underline{x}}\left(\underline{X}_{i}, Y_{i}\right)$ standing for $U_{n i}$. Applying Lemma 5.4 to (A.34) with $B_{1} \equiv M_{n}^{(1)}, B_{2} \equiv C n h^{d}\left(M_{n}^{(1)}\right)^{2} M_{n}^{(2)} l_{n} / h, \eta \equiv M^{3 / 2} d_{n} / 36$ and $r_{n}=r(n)$, we have (note that $n B_{1} / \eta \rightarrow \infty$ indeed)

$$
\lambda_{n} \eta / 4=C M^{1 / 2} \log n, \lambda_{n}^{2} B_{2}=C r_{n}^{-2 / \nu_{2}} \log n=o\left(\lambda_{n} \eta\right) .
$$

Thus, $\mathrm{T}_{n} \Psi_{n}$ is again summable over $n$ and (A.34) indeed holds.

Proof of Theorem 3.2. Let $\lambda_{1}=\lambda(s)$. Then according to Lemma 5.1 and Lemma 5.9, we know that with probability 1 , there exists some $C_{1}>1$, such that for all large $M>0$,

$$
\begin{aligned}
& \sup _{\substack{x \in \mathcal{D} \\
\sup _{\alpha \in B_{n}^{(1)}}^{(1)}, \beta \in B_{n}^{(2)}}}\left|\sum_{i=1}^{n} \Phi_{n i}(\underline{x} ; \alpha, \beta)-\frac{n h^{d}}{2}\left(H_{n} \alpha\right)^{\top} S_{n p}(\underline{x}) H_{n}(\alpha+2 \beta)\right| \\
& \leq C_{1} M^{3 / 2}\left(d_{n 1}+d_{n}\right) \leq 2 C_{1} M^{3 / 2}\left(n h^{d}\right)^{1-2 \lambda_{1}}(\log n)^{2 \lambda_{1}} \text { for large } n,
\end{aligned}
$$

where $d_{n 1}=\left(n h^{d}\right)^{1-\lambda_{1}-2 \lambda_{2}}(\log n)^{\lambda_{1}+2 \lambda_{2}}$. Note that based on (12), we can write

$$
\sum_{i=1}^{n} K_{n i} \varphi\left(Y_{i} ; \mu_{n i}^{\top} \beta_{p}(\underline{x})\right) \mu_{n i}^{\top} \alpha=n h^{d} \beta_{n}^{*}(\underline{x})^{\top} W_{p}^{-1} S_{n p}(\underline{x}) H_{n} \alpha .
$$


Replace $B_{n}^{(1)}$ in (A.35) with $B_{n k}^{(1)}=\left\{\alpha \in \mathcal{R}^{N}: k \leq M^{-1}\left(n h^{d} / \log n\right)^{\lambda_{1}}\left|H_{n} \alpha\right| \leq k+1\right\}$ and $M$ with $(k+1) M$. We have, by the definition of $\Phi_{n i}(\underline{x} ; \alpha, \beta)$, that

$$
\begin{aligned}
& \inf _{\underline{x} \in \mathcal{D}} \inf _{\substack{\alpha \in B_{n k}^{(1)} \\
\beta \in B_{n}^{(2)}}}\left\{\sum_{i=1}^{n} \rho\left(Y_{i} ; \mu_{n i}^{\top}\left(\alpha+\beta+\beta_{p}(\underline{x})\right)\right) K_{n i}-\sum_{i=1}^{n} \rho\left(Y_{i} ; \mu_{n i}^{\top}\left(\beta+\beta_{p}(\underline{x})\right)\right) K_{n i}\right. \\
&\left.+n h^{d}\left(W_{p}^{-1} \beta_{n}^{*}(\underline{x})-H_{n} \beta\right)^{\top} S_{n p}(\underline{x}) H_{n} \alpha\right\} \\
& \geq \quad \inf _{\underline{x} \in \mathcal{D}} \inf _{\alpha \in B_{n k}^{(1)}} \frac{n h^{d}}{2}\left(H_{n} \alpha\right)^{\top} S_{n p}(\underline{x}) H_{n} \alpha-2 C M^{3 / 2}\left(n h^{d}\right)^{1-2 \lambda_{1}}(\log n)^{2 \lambda_{1}} \\
& \geq \quad\left\{C_{3}(k M)^{2} / 2-2 C_{1}(k+1)^{3 / 2} M^{3 / 2}\right\}\left(n h^{d}\right)^{1-2 \lambda_{1}}(\log n)^{2 \lambda_{1}} \\
& \geq \quad\left(8-2^{5 / 2}\right) C_{1} C_{4}^{3 / 2}\left(n h^{d}\right)^{1-2 \lambda_{1}}(\log n)^{2 \lambda_{1}}>0 \text { almost surely, }
\end{aligned}
$$

where the last term is independent of the choice of $k \geq 1$. The last inequality is derived as follows. As $S_{p}>0$, suppose its minimum eigenvalue is $\tau_{1}>0$. As $S_{n p}(\underline{x}) \rightarrow g(\underline{x}) f(\underline{x}) S_{p}$ uniformly in $\underline{x} \in \mathcal{D}$ by Lemma 5.8 and $g(\underline{x}) f(\underline{x})$ is bounded away from zero by (A5) and (A.7), there exists some constant $C_{3}>0$, such that for all $\underline{x} \in \mathcal{D}$, the minimum eigenvalue of $S_{n p}(\underline{x})$ is greater than $C_{3}$. The last inequality thus holds if $M \geq C_{4}=\left(16 C_{1} / C_{3}\right)^{2}$. Note that

$$
\bigcup_{k=1}^{\infty} B_{n k}^{(1)}=\left\{\alpha\left|\in \mathcal{R}^{N}:\left(\frac{n h^{d}}{\log n}\right)^{\lambda_{1}}\right| H_{n} \alpha \mid \geq M\right\}:=B_{n}^{N} .
$$

Therefore, from (A.36) and (A.37), we have

$$
\begin{aligned}
\inf _{\underline{x} \in \mathcal{D}} & \inf _{\substack{\alpha \in B_{n}^{N} \\
\beta \in B_{n}^{(2)}}}\left\{\sum_{i=1}^{n} \rho\left(Y_{i} ; \mu_{n i}^{\top}\left(\alpha+\beta+\beta_{p}(\underline{x})\right)\right) K_{n i}-\sum_{i=1}^{n} \rho\left(Y_{i} ; \mu_{n i}^{\top}\left(\beta+\beta_{p}(\underline{x})\right)\right) K_{n i}\right. \\
& \left.+n h^{d}\left(W_{p}^{-1} \beta_{n}^{*}(\underline{x})-H_{n} \beta\right)^{\top} S_{n p}(\underline{x}) H_{n} \alpha\right\}>0 \text { almost surely. }
\end{aligned}
$$

Note that by (A.40), Lemma 5.10 and Proposition 3.1, we have $\left|\beta_{n}^{*}(\underline{x})\right| \leq C_{3}\left(n h^{d} / \log n\right)^{-\lambda_{2}}$ uniformly in $\underline{x} \in \mathcal{D}$ almost surely. Namely, $\beta_{n}^{*}(\underline{x}) \in B_{n}^{(2)}$ for all $\underline{x} \in \mathcal{D}$, if $M>C_{3}^{4}$. This implies that if $M>\max \left(C_{3}^{4}, C_{4}\right)$, (A.38) still holds with $\beta$ replaced with $H_{n}^{-1} W_{p}^{-1} \beta_{n}^{*}(\underline{x})$. Therefore,

$$
\begin{aligned}
\inf _{\underline{x} \in \mathcal{D}} \inf _{\alpha \in B_{n}^{N}}\left\{\sum_{i=1}^{n} K_{n i} \rho\left(Y_{i} ; \mu_{n i}^{\top}\left(\alpha+H_{n}^{-1} W_{p}^{-1} \beta_{n}^{*}(\underline{x})+\beta_{p}(\underline{x})\right)\right)\right. & \\
& \left.-\sum_{i=1}^{n} K_{n i} \rho\left(Y_{i} ; \mu_{n i}^{\top}\left(H_{n}^{-1} W_{p}^{-1} \beta_{n}^{*}(\underline{x})+\beta_{p}(\underline{x})\right)\right)\right\}>0,
\end{aligned}
$$


which is equivalent to Theorem 3.2.

Proof of (13). Let $\tilde{d}_{n}=\left(n h^{d}\right)^{1-2 \lambda_{1}}(\log n)^{2 \lambda_{1}}$. Following the proof lines of Theorem 3.2, we can see that (13) will follow if

$$
\sup _{\substack { x \in \mathcal{D} \\
\begin{subarray}{c}{\alpha \in B_{n}^{(1)} \\
\beta \in B_{n}^{(2)}{ x \in \mathcal { D } \\
\begin{subarray} { c } { \alpha \in B _ { n } ^ { ( 1 ) } \\
\beta \in B _ { n } ^ { ( 2 ) } } }\end{subarray}}\left|\sum_{i=1}^{n} R_{n i}(\underline{x} ; \alpha, \beta)\right| \leq M^{3 / 2} \tilde{d}_{n} \text { almost surely, }
$$

with $\lambda_{1}=1, \lambda_{2}=1 / 2$ and $B_{n}^{(i)}, i=1,2$ defined as in Lemma 5.1.

To prove this, cover $\mathcal{D}$ by a finite number $\tilde{\mathrm{T}}_{n}=\left\{\left(n h^{d} / \log n\right)^{1 / 2} / h\right\}^{d}$ of cubes $\mathcal{D}_{k}=\mathcal{D}_{n k}$ with side length $\tilde{l}_{n}=O\left\{h\left(n h^{d} / \log n\right)^{-1 / 2}\right\}$ and centers $\underline{x}_{k}=\underline{x}_{n, k}$. Write

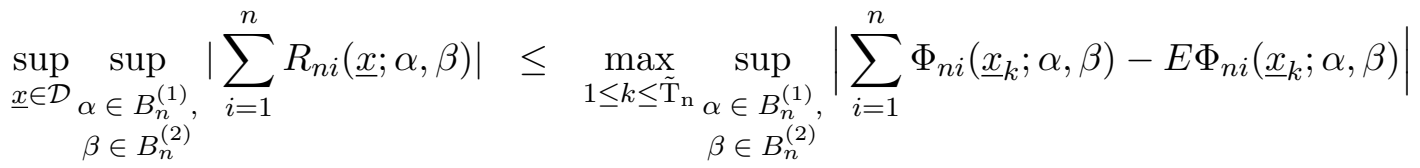

$$
\begin{aligned}
& +\max _{1 \leq k \leq \tilde{\mathrm{T}}_{n}} \sup _{\underline{x} \in \mathcal{D}_{k}} \sup _{\substack{\alpha \in B_{n}^{(1)} \\
\beta \in B_{n}^{(2)}}}\left|\sum_{i=1}^{n}\left\{\Phi_{n i}\left(\underline{x}_{k} ; \alpha, \beta\right)-\Phi_{n i}(\underline{x} ; \alpha, \beta)\right\}\right|
\end{aligned}
$$

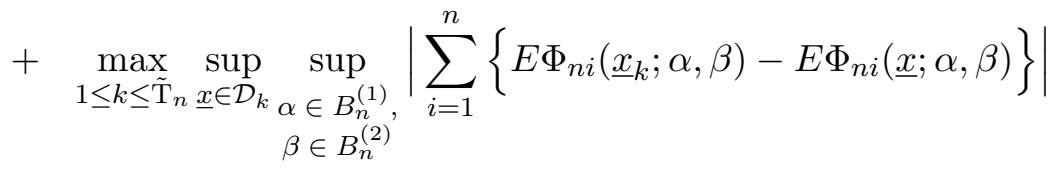

$$
\begin{aligned}
& \equiv Q_{1}+Q_{2}+Q_{3} \text {. }
\end{aligned}
$$

We will show that with probability $1, Q_{k} \leq M^{3 / 2} \tilde{d}_{n} / 3, k=1,2,3$.

Define $\xi_{i j}$ as in Lemma 5.1. As $P\left(Q_{2}>M^{3 / 2} \tilde{d}_{n} / 2\right) \leq \tilde{\mathrm{T}}_{n}\left(P_{n 1}+P_{n 2}+P_{n 3}\right)$, where

$$
P_{n j} \equiv \max _{1 \leq k \leq \tilde{T}_{n}} P\left(\sup _{\substack{x \in \mathcal{D}_{k} \\ \sup _{\alpha \in B_{n}^{(1)}}, \beta \in B_{n}^{(2)}}}\left|\sum_{i=1}^{n} \xi_{i j}\right| \geq M^{3 / 2} \tilde{d}_{n} / 9\right), j=1,2,3 .
$$

Then by Borel-Cantelli lemma, $Q_{2} \leq M^{3 / 2} \tilde{d}_{n} / 2$ almost surely, if $\sum_{n} \tilde{\mathrm{T}}_{n} P_{n j}<\infty$, for $j=1,2,3$. We only prove that for $P_{n 1}$ to illustrate. Recall that

$$
\xi_{i 1}=\left(K_{i k} \mu_{i k}-K_{i x} \mu_{i x}\right)^{\top} \alpha \int_{0}^{1}\left\{\varphi_{n i}\left(\underline{x}_{k} ; \mu_{i k}^{\top}(\beta+\alpha t)\right)-\varphi_{n i}\left(\underline{x}_{k} ; 0\right)\right\} d t
$$

Because $\left|\left(K_{i k} \mu_{i k}-K_{i x} \mu_{i x}\right)^{\top} \alpha\right| \leq C_{2} M_{n}^{(1)} \tilde{l}_{n} / h,\left|\mu_{i k}^{\top}(\beta+\alpha t)\right| \leq C M_{n}^{(2)}$ and $\varphi($.$) is Lipschitz$ continuous, we have $\left|\xi_{i 1}\right| \leq C M_{n}^{(2)} M_{n}^{(1)} \tilde{l}_{n} / h$. Define $U_{i k}=I\left\{\left|\underline{X}_{i k}\right| \leq 2 h\right\}$. As $\tilde{l}_{n}=o(h)$, we can 
see that $\xi_{i 1}=\xi_{i 1} U_{i k}$ and similar to (A.18), we have

$$
\begin{aligned}
& P\left(\sup _{\substack{\alpha \in B_{n}^{(1)}, \underline{x} \in \mathcal{D}_{k} \\
\beta \in B_{n}^{(2)}}} \sup _{i=1}\left|\xi_{i 1}\right|>\frac{M^{3 / 2} \tilde{d}_{n}}{9}\right) \leq P\left(\sum_{i=1}^{n} U_{i k}>\frac{M^{1 / 4} n h^{d}}{9 C}\right) \\
& \leq P\left(\left|\sum_{i=1}^{n} U_{i k}-E U_{i k}\right|>\frac{M^{1 / 4} n h^{d}}{18 C}\right),
\end{aligned}
$$

and $\sum_{n} \tilde{\mathrm{T}}_{n} P_{n j}<\infty$ thus follows from similar arguments as those lying between (A.18) and (A.19).

The proof of $Q_{1} \leq M^{3 / 2} \tilde{d}_{n} / 2$ almost surely is much easier than in Lemma 5.1, if $\varphi($.$) is Lipschitz$ continuous. Instead of the iterative partition approach adopted there, we once for all partition $B_{n}^{(i)}, i=1,2$, into a sequence of disjoint subrectangles $D_{1}^{(i)}, \cdots, D_{J_{1}}^{(i)}$ such that

$$
\left|D_{j_{1}}^{(i)}\right|=\sup \left\{\left|H_{n}(\alpha-\beta)\right|: \alpha, \beta \in D_{j_{1}}^{(i)}\right\} \leq M_{n}^{(i)}(\log n / n)^{1 / 2}, \quad 1 \leq j_{1} \leq J_{1}
$$

Obviously $J_{1} \leq(n / \log n)^{N / 2}$. Choose a point $\alpha_{j_{1}} \in D_{j_{1}}^{(1)}$ and $\beta_{k_{1}} \in D_{k_{1}}^{(2)}$. Then

$$
\begin{aligned}
Q_{1} \leq & \max _{\substack{1 \leq k \leq \tilde{\mathrm{T}}_{n} \\
1 \leq j_{1}, k_{1} \leq J_{1}}} \sup _{\substack{\alpha \in D_{j_{1}}^{(1)}, \beta \in D_{k_{1}}^{(2)}}}\left|\sum_{i=1}^{n}\left\{R_{n i}\left(\underline{x}_{k} ; \alpha_{j_{1}}, \beta_{k_{1}}\right)-R_{n i}\left(\underline{x}_{k} ; \alpha, \beta\right)\right\}\right| \\
& +\max _{\substack{1 \leq k \leq \mathrm{T}_{n} \\
1 \leq j_{1}, k_{1} \leq J_{1}}}\left|\sum_{i=1}^{n} R_{n i}\left(\underline{x}_{k} ; \alpha_{j_{1}}, \beta_{k_{1}}\right)\right|=H_{n 1}+H_{n 2} .
\end{aligned}
$$

By Lipschitz continuity of $\varphi($.$) , we have for any \alpha \in D_{j_{1}}^{(1)}$ and $\beta \in D_{k_{1}}^{(2)}$,

$$
\left|\Phi_{n i}\left(\underline{x}_{k} ; \alpha_{j_{1}}, \beta_{k_{1}}\right)-\Phi_{n i}\left(\underline{x}_{k} ; \alpha, \beta\right)\right|^{2}=O\left(\left\{M_{n}^{(2)}\right\}^{3} \log n / n\right)<M^{3 / 2} \tilde{d}_{n} /(4 n) .
$$

Therefore, it remains to show that $P\left(H_{n 2}>M^{3 / 2} \tilde{d}_{n} / 4\right)$ is summable over $n$.

First note that by Cauchy inequality, $\left|R_{n i}(\underline{x} ; \alpha, \beta)\right|^{2}=O\left(\left\{M_{n}^{(1)} M_{n}^{(2)}\right\}^{2}\right)$ and $E\left|R_{n i}(\underline{x} ; \alpha, \beta)\right|^{2}=$ $O\left(h^{d}\left\{M_{n}^{(1)} M_{n}^{(2)}\right\}^{2}\right)$ uniformly in $\underline{X}_{i}, \underline{x}, \alpha \in M_{n}^{(1)}$ and $\beta \in M_{n}^{(2)}$. Next, for any $\eta>0$,

$$
P\left(H_{n 2}>\eta\right) \leq \tilde{\mathrm{T}}_{n} J_{1}^{2} P\left(\left|\sum_{i=1}^{n} R_{n i}\left(\underline{x} ; \alpha_{j_{1}}, \beta_{k_{1}}\right)\right|>\eta\right) .
$$

We apply Lemma 5.4 with $r_{n}=\left(n h^{d} / \log n\right)^{1 / 2}, B_{1}=2 C_{1} M_{n}^{(1)} M_{n}^{(2)}, B_{2}=C_{2} n h^{d}\left(M_{n}^{(1)} M_{n}^{(2)}\right)^{2}, \lambda_{n}=$ 
$\left(4 C_{1} r_{n}\left\{M_{n}^{(2)}\right\}^{2}\right)^{-1}$ and $\eta=M^{3 / 2} \tilde{d}_{n} / 4$. It is easy to see that $n B_{1} / \eta \rightarrow \infty$ and

$$
\begin{aligned}
& \lambda_{n} \eta / 4=M \log n /\left(16 C_{1}\right), \lambda_{n}^{2} B_{2}=o\left(\lambda_{n} \eta\right) \\
& \Psi(n) \equiv q_{n}\left\{n B_{1} / \eta\right\}^{1 / 2} \gamma\left[r_{n}\right]=n^{3 / 2}(\log n)^{-1 / 2} \gamma[r(n)] / r(n) .
\end{aligned}
$$

As $\tilde{\mathrm{T}}_{n} J_{1}^{2} \Psi(n)$ is summable over $n$ by condition (A.4), so is $P\left(H_{n 2}>M^{3 / 2} \tilde{d}_{n} / 4\right)$.

Proof of Corollary 3.3. As $1+\lambda_{2} \geq 2 \lambda_{1}$, it's sufficient to prove that with probability 1 ,

$$
\beta_{n}^{*}(\underline{x})-E \beta_{n}^{*}(\underline{x})-\frac{1}{n h^{d}} W_{p} S_{n p}^{-1}(\underline{x}) H_{n}^{-1} \sum_{i=1}^{n} K_{h}\left(\underline{X}_{i}-\underline{x}\right) \varphi\left(\varepsilon_{i}\right) \mu\left(\underline{X}_{i}-\underline{x}\right)=O\left\{\left(\frac{\log n}{n h^{d}}\right)^{\left(1+\lambda_{2}\right) / 2}\right\},
$$

uniformly in $\underline{x} \in \mathcal{D}$. As $\varphi\left(\varepsilon_{i}\right) \equiv \varphi\left(Y_{i}, m\left(X_{i}\right)\right)$ and $E \varphi\left(\varepsilon_{i}\right)=0$, the term on the left hand side of (A.40) stands for

$$
W_{p} S_{n, p}^{-1}(\underline{x}) \frac{1}{n h^{d}} \sum_{i=1}^{n}\left\{Z_{n i}(\underline{x})-E Z_{n i}(\underline{x})\right\}
$$

where

$$
Z_{n i}(\underline{x})=H_{n}^{-1} K_{h}\left(\underline{X}_{i}-\underline{x}\right) \mu\left(\underline{X}_{i}-\underline{x}\right)\left\{\varphi\left(Y_{i}, \mu\left(\underline{X}_{i}-\underline{x}\right)^{\top} \beta_{p}(\underline{x})\right)-\varphi\left(\varepsilon_{i}\right)\right\} .
$$

Next, similar to what we did in Lemma 5.1 , we cover $\mathcal{D}$ with number $\mathrm{T}_{n}$ cubes $\mathcal{D}_{k}=\mathcal{D}_{n, k}$ with side length $l_{n}=O\left(\mathrm{~T}_{n}^{-1 / d}\right)$ and centers $\underline{x}_{k}=\underline{x}_{n, k}$. Write

$$
\begin{aligned}
\sup _{\underline{x} \in \mathcal{D}}\left|\sum_{i=1}^{n} Z_{n i}(\underline{x})-E Z_{n i}(\underline{x})\right| \leq & \max _{1 \leq k \leq \mathrm{T}_{\mathrm{n}}}\left|\sum_{i=1}^{n} Z_{n i}\left(\underline{x}_{k}\right)-E Z_{n i}\left(\underline{x}_{k}\right)\right| \\
& +\max _{1 \leq k \leq \mathrm{T}_{n}} \sup _{\underline{x} \in \mathcal{D}_{k}}\left|\sum_{i=1}^{n} Z_{n i}(\underline{x})-Z_{n i}\left(\underline{x}_{k}\right)\right| \\
& +\max _{1 \leq k \leq \mathrm{T}_{n}} \sup _{\underline{x} \in \mathcal{D}_{k}}\left|\sum_{i=1}^{n} E Z_{n i}(\underline{x})-E Z_{n i}\left(\underline{x}_{k}\right)\right| \\
\equiv & Q_{1}+Q_{2}+Q_{3} .
\end{aligned}
$$

As $Z_{n i}(\underline{x})-Z_{n i}\left(\underline{x}_{k}\right)=H_{n}^{-1} K_{h}\left(\underline{X}_{i}-\underline{x}\right) \mu\left(\underline{X}_{i}-\underline{x}\right)\left\{\varphi_{n i}(\underline{x} ; 0)-\varphi_{n i}\left(\underline{x}_{k} ; 0\right)\right\}$, through approaches similar to that for $\xi_{i 3}$ in the proof of Lemma 5.2, we can show that

$$
Q_{2}=O\left\{\left(\frac{n h^{d}}{\log n}\right)^{\left(1-\lambda_{2}\right) / 2} \log n\right\} \text { almost surely }
$$

and the same result for $Q_{3}$ also holds. To bound $Q_{1}$, first note that $E Z_{n i}^{2}\left(\underline{x}_{k}\right)=O\left(h^{p+1+d}\right)$ uniformly in $i$ and $k$. As $\left|Z_{n i}(\underline{x})\right| \leq C$ for some constant $C$ by (A2), we can see that from 
Lemma 5.5

$$
\sum_{i=1}^{n} E Z_{n i}^{2}\left(\underline{x}_{k}\right)+\sum_{i<j}\left|\operatorname{Cov}\left(Z_{n i}\left(\underline{x}_{k}\right), Z_{n j}\left(\underline{x}_{k}\right)\right)\right| \leq C_{2} n h^{p+1+d} .
$$

Finally by Lemma 5.4 with $B_{1}=C_{1}, B_{2} \equiv C n h^{p+1+d}, \eta=A_{3}\left(n h^{d} / \log n\right)^{\left(1-\lambda_{2}\right) / 2} \log n$ and $r_{n}=r(n)$, we have, as $n B_{1} / \eta \rightarrow \infty$ that

$$
\lambda_{n} \eta=A_{3} /\left(2 C_{1}\right) \log n, \lambda_{n}^{2} B_{2}=C_{2} /\left(4 C_{1}^{2}\right) \log n .
$$

Therefore,

$$
P\left(\max _{1 \leq k \leq \mathrm{T}_{\mathrm{n}}}\left|\sum_{i=1}^{n} Z_{n i}\left(\underline{x}_{k}\right)-E Z_{n i}\left(\underline{x}_{k}\right)\right| \geq A_{3}\left(n h^{d} / \log n\right)^{\left(1-\lambda_{2}\right) / 2} \log n\right) \leq \mathrm{T}_{n} / n^{a}+C \mathrm{~T}_{n} \Psi_{n},
$$

where $a=A_{3} /\left(8 C_{1}\right)-C_{2} /\left(4 C_{1}^{2}\right)$. By selecting $A_{3}$ large enough, we can ensure that $\mathrm{T}_{n} / n^{a}$ is summable over $n$. As $\mathrm{T}_{n} \Psi_{n}$ is summable over $n$ from (A.4), we can conclude that

$$
Q_{1}=O\left\{\left(\frac{n h^{d}}{\log n}\right)^{\left(1-\lambda_{2}\right) / 2} \log n\right\} \text { almost surely. }
$$

This together with Lemma 5.8 completes the proof.

Proof of Corollary 4.1. Through the proof lines for Theorem 3.2 and Corollary 3.3, it's not difficult to see that Corollary 3.3 still holds under the conditions imposed here. Under the additive structure (4), we thus have

$$
\begin{aligned}
\phi_{n 1}\left(x_{1}\right)= & \phi_{1}\left(x_{1}\right)+\frac{1}{n} \sum_{i=1}^{n} m_{2}\left(\underline{X}_{2 i}\right)-h^{p+1} e_{1} W_{p} S_{p}^{-1} B_{1} \frac{1}{n} \sum_{i=1}^{n} \mathbf{m}_{p+1}\left(x_{1}, \underline{X}_{2 i}\right) \\
& +\frac{1}{n^{2} h_{1} h^{d-1}} e_{1} \sum_{j=1}^{n} \varphi\left(\varepsilon_{j}\right) \sum_{i=1}^{n} S_{n p}^{-1}\left(x_{1}, \underline{X}_{2 i}\right) K\left(X_{1, x j} / h_{1}, \underline{X}_{2, i j} / h\right) \mu\left(X_{1, x j} / h_{1}, \underline{X}_{2, i j} / h\right) \\
& +o_{p}\left(\left\{\max \left(h_{1}, h\right)\right\}^{p+1}\right)+O_{p}\left\{\left(n h_{1} h^{d-1} / \log n\right)^{-3 / 4}\right\}
\end{aligned}
$$

where $X_{1, x j}=X_{1 j}-x, \underline{X}_{2, i j}=\underline{X}_{2 i}-\underline{X}_{2 j}$ and $e_{1}$ is as in Proposition 3.1. Note that by (17), $\left(n h_{1}\right)^{1 / 2}\left(n h_{1} h^{d-1} / \log n\right)^{-3 / 4} \rightarrow 0$, the $O_{p}($.$) term can thus be safely ignored.$

By central limit theorem for strongly mixing processes (Bosq, 1998, Theorem 1.7), we have

$$
\frac{1}{n} \sum_{i=1}^{n} m_{2}\left(\underline{X}_{2 i}\right)=O_{p}\left(n^{-1 / 2}\right), \quad \frac{1}{n} \sum_{i=1}^{n} \mathbf{m}_{p+1}\left(x_{1}, \underline{X}_{2 i}\right)=E \mathbf{m}_{p+1}\left(x_{1}, \underline{X}_{2}\right)+O_{p}\left(n^{-1 / 2}\right) .
$$


As the expectations of all other terms in (A.41) are 0, the leading term in the asymptotic bias of $\tilde{\phi}_{1}\left(x_{1}\right)-\phi_{1}\left(x_{1}\right)$ is thus given by

$$
-\left\{\max \left(h_{1}, h\right)\right\}^{p+1} e_{1} W_{p} S_{p}^{-1} B_{1} E \mathbf{m}_{p+1}\left(x_{1}, \underline{X}_{2}\right) .
$$

Again through standard arguments in Masry (1996), we can see that

$$
\begin{aligned}
& \frac{1}{n h^{d-1}} \sum_{i=1}^{n} S_{n p}^{-1}\left(x_{1}, \underline{X}_{2 i}\right) K_{h}\left(X_{1, x j}, \underline{X}_{2, i j}\right) \mu\left(X_{1, x j} / h_{1}, \underline{X}_{2, i j} / h\right) \\
= & S_{n p}^{-1}\left(x_{1}, \underline{X}_{2 j}\right) f_{2}\left(\underline{X}_{2 j}\right) \int_{[0,1] \otimes d-1}\{K \mu\}\left(X_{1, x j} / h_{1}, \underline{v}\right) d \underline{v}\left\{1+O\left(\left\{\frac{\log n}{n h^{d-1}}\right\}^{1 / 2}\right)\right\}
\end{aligned}
$$

uniformly in $1 \leq i \leq n$. Therefore, the leading term in the asymptotic variance of $\phi_{n 1}\left(x_{1}\right)-\phi_{1}\left(x_{1}\right)$ is the variance of the following term

$$
\left(n h_{1}\right)^{-1} e_{1} \sum_{j=1}^{n} \varphi\left(\varepsilon_{j}\right) S_{n p}^{-1}\left(x_{1}, \underline{X}_{2 j}\right) f_{2}\left(\underline{X}_{2 j}\right) \int_{[0,1] \otimes d-1}\{K \mu\}\left(X_{1, x j} / h_{1}, \underline{v}\right) d \underline{v},
$$

which is asymptotically

$$
\left(n h_{1}\right)^{-1}\left\{\int_{[0,1]^{\otimes d-1}}\left\{f g^{2}\right\}^{-1}\left(x_{1}, \underline{X}_{2}\right) f_{2}^{2}\left(\underline{X}_{2}\right) \sigma^{2}\left(x_{1}, \underline{X}_{2}\right) d \underline{X}_{2}\right\} e_{1} S_{p}^{-1} K_{2} K_{2}^{\top} S_{p}^{-1} e_{1}^{\top} .
$$

If $\rho(y ; \theta)=(2 q-1)(y-\theta)+|y-\theta|$ and $\varphi(\theta)=2 q I\{\theta>0\}+(2 q-2) I\{\theta<0\}$, we have $g(\underline{x})=2 f_{\varepsilon}(0 \mid \underline{x})$ and

$$
\sigma^{2}(\underline{x})=E\left[\varphi^{2}(\varepsilon) \mid \underline{X}=\underline{x}\right]=4 q^{2}\left(1-F_{\varepsilon}(0)\right)+4(1-q)^{2} F_{\varepsilon}(0)=4 q(1-q),
$$

which when substituted into (A.42), yields the asymptotic variance of the quantile regression estimator,

$\tilde{\sigma}^{2}\left(x_{1}\right)=q(1-q)\left\{\int_{[0,1] \otimes d-1} f^{-1}\left(x_{1}, \underline{X}_{2}\right) f_{\varepsilon}^{-2}\left(0 \mid x_{1}, \underline{X}_{2}\right) f_{2}^{2}\left(\underline{X}_{2}\right) d \underline{X}_{2}\right\} e_{1} S_{p}^{-1} K_{2} K_{2}^{\top} S_{p}^{-1} e_{1}^{\top}$.

The next Lemma is due to Davydov (Hall and Heyde (1980), Corollary A.2).

Lemma 5.3 Suppose $X$ and $Y$ are random variables which are respectively $\mathcal{G}-$ and $\mathcal{H}-$ measurable, where $\mathcal{G}$ - and $\mathcal{H}$ - are two $\sigma$-algebras. $E|X|^{p}<\infty, E|Y|^{q}<\infty$, with $p>1, q>1$, and $p^{-1}+q^{-1}<1$. Then

$$
|E X Y-E X E Y| \leq 8\|X\|_{p}\|Y\|_{q}\left\{\sup _{A \in \mathcal{G}, B \in \mathcal{H}}|P(A B)-P(A) P(B)|\right\}^{1-p^{-1}-q^{-1}} .
$$


The next lemma is a generalization of some results in the proof of Theorem 2 in Masry (1996).

Lemma 5.4 Suppose $\left\{Z_{i}\right\}_{i=1}^{\infty}$ is a zero-mean strictly stationary processes with strong mixing coefficient $\gamma[k]$, and that $\left|Z_{i}\right| \leq B_{1}, \sum_{i=1}^{n} E Z_{i}^{2}+\sum_{i<j}\left|\operatorname{Cov}\left(Z_{i}, Z_{j}\right)\right| \leq B_{2}$. Then for any $\eta>0$ and integer series $r_{n} \rightarrow \infty$, if $n B_{1} / \eta \rightarrow \infty$ and $q_{n} \equiv\left[n / r_{n}\right] \rightarrow \infty$, we have

$$
P\left(\left|\sum_{i=1}^{n} Z_{i}\right| \geq \eta\right) \leq 4 \exp \left\{-\frac{\lambda_{n} \eta}{4}+\lambda_{n}^{2} B_{2}\right\}+C \Psi(n)
$$

where $\Psi(n)=q_{n}\left\{n B_{1} / \eta\right\}^{1 / 2} \gamma\left[r_{n}\right], \lambda_{n}=1 /\left\{2 r_{n} B_{1}\right\}$.

Proof. We partition the set $\{1, \cdots, n\}$ into $2 q \equiv 2 q_{n}$ consecutive blocks of size $r \equiv r_{n}$ with $n=2 q r+v$ and $0 \leq v<r$. Write

$$
V_{n}(j)=\sum_{i=(j-1) r+1}^{j r} Z_{i}, j=1, \cdots, 2 q
$$

and

$$
W_{n}^{\prime}=\sum_{j=1}^{q} V_{n}(2 j-1), W_{n}^{\prime \prime}=\sum_{j=1}^{q} V_{n}(2 j), W_{n}^{\prime \prime \prime}=\sum_{i=2 q r+1}^{n} Z_{i} .
$$

Then $W_{n} \equiv \sum_{i=1}^{n} Z_{i}=W_{n}^{\prime}+W_{n}^{\prime \prime}+W_{n}^{\prime \prime \prime}$. The contribution of $W_{n}^{\prime \prime \prime}$ is negligible as it consists of at most $r$ terms compared of $q r$ terms in $W_{n}^{\prime}$ or $W_{n}^{\prime \prime}$. Then by the stationarity of the processes, for any $\eta>0$,

$$
P\left(W_{n}>\eta\right) \leq P\left(W_{n}^{\prime}>\eta / 2\right)+P\left(W_{n}^{\prime \prime}>\eta / 2\right)=2 P\left(W_{n}^{\prime}>\eta / 2\right) .
$$

To bound $P\left(W_{n}^{\prime}>\eta / 2\right)$, using recursively Bradley's Lemma, we can approximate the random variables $V_{n}(1), V_{n}(3), \cdots, V_{n}(2 q-1)$ by independent random variables $V_{n}^{*}(1), V_{n}^{*}(3), \cdots, V_{n}^{*}(2 q-$ $1)$, which satisfy that for $1 \leq j \leq q, V_{n}^{*}(2 j-1)$ has the same distribution as $V_{n}(2 j-1)$ and

$$
P\left(\left|V_{n}^{*}(2 j-1)-V_{n}(2 j-1)\right|>u\right) \leq 18\left(\left\|V_{n}(2 j-1)\right\|_{\infty} / u\right)^{1 / 2} \sup |P(A B)-P(A) P(B)|
$$

where $u$ is any positive value such that $0<u \leq\left\|V_{n}(2 j-1)\right\|_{\infty}<\infty$ and the supremum is taken over all sets of $A$ and $B$ in the $\sigma$-algebras of events generated by $\left\{V_{n}(1), V_{n}(3), \cdots, V_{n}(2 j-\right.$ 
$3)\}$ and $V_{n}(2 j-1)$ respectively. By the definition of $V_{n}(j)$, we can see that $\sup \mid P(A B)-$ $P(A) P(B) \mid=\gamma\left[r_{n}\right]$. Write

$$
\begin{aligned}
P\left(W_{n}^{\prime}>\frac{\eta}{2}\right) & \leq P\left(\left|\sum_{j=1}^{q} V_{n}^{*}(2 j-1)\right|>\frac{\eta}{4}\right)+P\left(\left|\sum_{j=1}^{q} V_{n}(2 j-1)-V_{n}^{*}(2 j-1)\right|>\frac{\eta}{4}\right) \\
& \equiv I_{1}+I_{2} .
\end{aligned}
$$

We bound $I_{1}$ as follows. Let $\lambda=1 /\left\{2 B_{1} r\right\}$. Since $\left|Z_{i}\right| \leq B_{1}, \lambda\left|V_{n}(j)\right| \leq 1 / 2$, then using the fact that $e^{x} \leq 1+x+x^{2} / 2$ holds for $|x| \leq 1 / 2$, we have

$$
E\left\{e^{ \pm \lambda V_{n}^{*}(2 j-1)}\right\} \leq 1+\lambda^{2} E\left\{V_{n}(j)\right\}^{2} \leq e^{\lambda^{2} E\left\{V_{n}^{*}(2 j-1)\right\}^{2}}
$$

By Markov inequality, (A.46) and the independence of the $\left\{V_{n}^{*}(2 j-1)\right\}_{j=1}^{q}$, we have

$$
\begin{aligned}
I_{1} & \leq e^{-\lambda \eta / 4}\left[E \exp \left(\lambda \sum_{j=1}^{q} V_{n}^{*}(2 j-1)\right)+E \exp \left(-\lambda \sum_{j=1}^{q} V_{n}^{*}(2 j-1)\right)\right] \\
& \leq 2 \exp \left(-\lambda \eta / 4+\lambda^{2} \sum_{j=1}^{q} E\left\{V_{n}^{*}(2 j-1)\right\}^{2}\right) \\
& \leq 2 \exp \left\{-\lambda \eta / 4+C_{2} \lambda^{2} B_{2}\right\} .
\end{aligned}
$$

We now bound the term $I_{2}$ in (A.45). Notice that

$$
I_{2} \leq \sum_{j=1}^{q} P\left(\left|V_{n}(2 j-1)-V_{n}^{*}(2 j-1)\right|>\frac{\eta}{4 q}\right) .
$$

If $\left\|V_{n}(2 j-1)\right\|_{\infty} \geq \eta /(4 q)$, substitute $\eta /(4 q)$ for $u$ in (A.44),

$$
I_{2} \leq 18 q\left\{\left\|V_{n}(2 j-1)\right\| / \eta /(4 q)\right\}^{1 / 2} \gamma\left[r_{n}\right] \leq C q^{3 / 2} / \eta^{1 / 2} \gamma\left[r_{n}\right]\left(r_{n} B_{1}\right)^{1 / 2}
$$

If $\left\|V_{n}(2 j-1)\right\|_{\infty}<\eta /(4 q)$, let $u \equiv\left\|V_{n}(2 j-1)\right\|_{\infty}$ in (A.44) and we have

$$
I_{2} \leq C q \gamma\left[r_{n}\right]
$$

which is of smaller order than (A.48), if $n B_{1} / \eta \rightarrow \infty$. Thus by (A.43), (A.45), (A.47) and (A.48),

$$
P\left(W_{n}>\eta\right) \leq 4 \exp \left\{-\lambda_{n} \eta / 4+C_{2} B_{2} \lambda_{n}^{2}\right\}+C \Psi_{n}
$$

where the constant $C$ is independent of $n$. 
Lemma 5.5 For any $\underline{x} \in R^{d}$, let $\psi_{\underline{x}}\left(\underline{X}_{i}, Y_{i}\right)=I\left(\left|\underline{X}_{i x}\right| \leq h\right) \psi_{x}\left(\underline{X}_{i x}, Y_{i}\right)$, a measurable function of $\left(\underline{X}_{i}, Y_{i}\right)$ with $\left|\psi_{\underline{x}}\left(\underline{X}_{i}, Y_{i}\right)\right| \leq B$ and $V=E \psi_{\underline{x}}^{2}\left(\underline{X}_{i}, Y_{i}\right)$. Suppose the mixing coefficient $\gamma[k]$ satisfies (A.3). Then

$$
\operatorname{Cov}\left(\sum_{i=1}^{n}\left|\underline{x}_{\underline{x}}\left(\underline{X}_{i}, Y_{i}\right)\right|\right)=n V\left[1+o\left\{\left(B^{2} h^{p+d+1} / V\right)^{1-2 / \nu_{2}}\right\}\right] .
$$

Proof. Denote $\psi_{\underline{x}}\left(\underline{X}_{i}, Y_{i}\right)$ by $\psi_{i x}$. First note that

$$
\begin{aligned}
V=E \psi_{i x}^{2} & =h^{d} \int_{|\underline{u}| \leq 1} E\left(\psi_{i x}^{2} \mid \underline{X}_{i}=\underline{x}+h \underline{u}\right) f(\underline{x}+h \underline{u}) d \underline{u}, \\
\sum_{i<j}\left|\operatorname{Cov}\left(\psi_{i x}, \psi_{j x}\right)\right| & =\sum_{l=1}^{n-d}(n-l-d+1)\left|\operatorname{Cov}\left(\psi_{0 x}, \psi_{l x}\right)\right| \leq n \sum_{l=1}^{n-d}\left|\operatorname{Cov}\left(\psi_{0 x}, \psi_{l x}\right)\right| \\
& =n \sum_{l=1}^{d-1}+n \sum_{l=d}^{\pi_{n}}+n \sum_{l=\pi_{n}+1}^{n-d} \equiv n J_{21}+n J_{22}+n J_{23},
\end{aligned}
$$

where $\pi_{n}=h^{(p+d+1)\left(2 / \nu_{2}-1\right) / a}$. For $J_{21}$, there might be an overlap between the components of $\underline{X}_{0}$ and $\underline{X}_{l}$, for example, when $\underline{X}_{i}=\left(X_{i-d}, \cdots, X_{i-1}\right)$, where $\left\{X_{i}\right\}$ is a univariate time series. Without loss of generality, let $\underline{u}^{\prime}, \underline{u}^{\prime \prime}$ and $\underline{u}^{\prime \prime \prime}$ of dimensions $l, d-l$ and $l$ respectively, be the $d+l$ distinct random variables in $\left(\underline{X}_{0 x} / h, \underline{X}_{l x} / h\right)$. Write $\underline{u}_{1}=\left(\underline{u}^{\prime \top}, \underline{u}^{\prime \top \top}\right)^{\top}$ and $\underline{u}_{2}=\left(\underline{u}^{\prime \prime \top}, \underline{u}^{\prime \prime \prime \top}\right)^{\top}$. Then by Cauchy inequality, we have

$$
\left|E\left(\psi_{0 x},\left.\psi_{l x}\right|_{\underline{X}_{l}=\underline{x}+h \underline{u}_{1}}=\underline{\underline{x}}+\underline{u}_{2}\right)\right| \leq\left\{E\left(\psi_{0 x}^{2} \mid \underline{X}_{0}=\underline{x}+h \underline{u}_{1}\right) E\left(\psi_{j x}^{2} \mid \underline{X}_{j}=\underline{x}+h \underline{u}_{2}\right)\right\}^{1 / 2}=V / h^{d}
$$

and through a transformation of variables, we have

$\left|\operatorname{Cov}\left(\psi_{0 x}, \psi_{l x}\right)\right| \leq h^{l} V \int_{\left|\underline{u}_{1}\right| \leq 1}\left|f\left(\underline{x}+h \underline{u}_{1}, \underline{x}+h \underline{u}_{2} ; l\right)-f\left(\underline{x}+h \underline{u}_{1}\right) f\left(\underline{x}+h \underline{u}_{2} ; l+d-1\right)\right| d \underline{u}^{\prime} d \underline{u}^{\prime \prime} d \underline{u}^{\prime \prime \prime}$, where by (A4) and (A5), the integral is bounded. Therefore,

$$
n J_{21} \leq C n V \sum_{l=1}^{d-1} h^{l}=o(n V) .
$$

For $J_{22}$, there is no overlap between the components of $\underline{X}_{0}$ and $\underline{X}_{l}$. Let $\underline{X}_{0 x}=h \underline{u}$ and $\underline{X}_{l x}=h \underline{v}$ 
and we have

$$
\begin{aligned}
& \left|\operatorname{Cov}\left(\psi_{0 x}, \psi_{l x}\right)\right| \leq h^{2 d} \int_{\substack{|\underline{u}| \leq 1 \\
|\underline{v}| \leq 1}} E\left(\psi_{0 x},\left.\psi_{l x}\right|_{\underline{X}_{l}=\underline{x}+h \underline{\underline{x}}}\right) d \underline{u} d \underline{v} \\
& \times[f(\underline{x}+h \underline{u}, \underline{x}+h \underline{v} ; l+d-1)-f(\underline{x}+h \underline{u}) f(\underline{x}+h \underline{v})] \\
& =C h^{d} V
\end{aligned}
$$

where the last equality follows from (A4), (A5) and (A.49). Therefore, as $\pi_{n} h^{d} \rightarrow 0$,

$$
n J_{22}=O\left\{n \pi_{n} h^{d} V\right\}=o(n V)
$$

For $J_{23}$, using Davydov's lemma (Lemma 5.3) we have

$$
\left|\operatorname{Cov}\left(\psi_{0 x}, \psi_{l x}\right)\right| \leq 8\{\gamma[l-d+1]\}^{1-2 / \nu_{2}}\left\{E\left|\psi_{i x}\right|^{\nu_{2}}\right\}^{2 / \nu_{2}}, \text { as } \nu_{2}>2
$$

As $\left|\psi_{i x}\right| \leq B, E\left|\Phi_{n i}\right|^{\nu_{2}} \leq B^{\nu_{2}-2} V$

$$
J_{23} \leq C B^{(\nu-2) 2 / \nu_{2}} V^{2 / \nu_{2}} / \pi_{n}^{a} \sum_{l=\pi_{n}+1}^{\infty} l^{a}\{\gamma[l-d+1]\}^{1-2 / \nu_{2}},
$$

where the summation term is $o(1)$, as $\pi_{n} \rightarrow \infty$. Thus $J_{23}=o\left\{V\left(B^{2} h^{p+d+1} / V\right)^{1-2 / \nu_{2}}\right\}$, which completes the proof.

Lemma 5.6 Suppose (A2)- (A6) hold. Then for $U_{n i}^{l}, l=1, \cdots, m$ defined in (A.32) and $Z_{n i}, l=$ $1, \cdots, \mathrm{L}_{n}$ defined in (A.13), we have

$$
\begin{aligned}
& \sum_{i=1}^{n} E\left(U_{n i}^{l}\right)^{2}+\sum_{i<j}\left|\operatorname{Cov}\left(U_{n i}^{l}, U_{n j}^{l}\right)\right| \leq C n h^{d} M_{n}^{(1)}\left\{M_{n}^{(2)} / M_{n}^{(1)}\right\}^{1-2 / \nu_{2}}, \\
& \sum_{i=1}^{n} E Z_{n i}^{2}+\sum_{i<j}\left|\operatorname{Cov}\left(Z_{n i}, Z_{n j}\right)\right|=n h^{d}\left(M_{n}^{(1)}\right)^{2} M_{n}^{(2)}\left\{M^{l} \log n\right\}^{-2 / \nu_{2}}
\end{aligned}
$$

uniformly in $\underline{x}_{k}, 1 \leq k \leq \mathrm{T}_{n}$.

Proof. We only prove (A.52), which is more involved than (A.51). To simplify the notations, denote $\alpha_{j_{l}}, \beta_{k_{l}}, \alpha_{j_{l}}$ and $\beta_{j_{l}}$ by $\alpha_{1}, \beta_{1}, \alpha_{2}$ and $\beta_{2}$, respectively. Clearly,

$$
\int_{\underline{u}^{\top} H_{n} \beta_{2}}^{\underline{u}^{\top} H_{n}\left(\alpha_{2}+\beta_{2}\right)}\left\{\varphi_{n i}\left(\underline{x}_{k} ; t\right)-\varphi_{n i}\left(\underline{x}_{k} ; 0\right)\right\} d t=\int_{\underline{u}^{\top} H_{n} \beta_{1}}^{\underline{u}^{\top} H_{n}\left(\alpha_{2}+\beta_{1}\right)}\left\{\varphi_{n i}\left(\underline{x}_{k} ; t+\underline{u}^{\top} H_{n}\left(\beta_{2}-\beta_{1}\right)\right)-\varphi_{n i}\left(\underline{x}_{k} ; 0\right)\right\} d t,
$$


and

$$
\begin{aligned}
Z_{n i}= & \int_{\underline{u}^{\top} H_{n} \beta_{1}}^{\underline{u}^{\top} H_{n}\left(\alpha_{1}+\beta_{1}\right)}\left\{\varphi_{n i}\left(\underline{x}_{k} ; t\right)-\varphi_{n i}\left(\underline{x}_{k} ; 0\right)\right\} d t-\int_{\underline{u}^{\top} H_{n} \beta_{2}}^{u^{\top} H_{n}\left(\alpha_{2}+\beta_{2}\right)}\left\{\varphi_{n i}\left(\underline{x}_{k} ; t\right)-\varphi_{n i}\left(\underline{x}_{k} ; 0\right)\right\} d t \\
= & \int_{\underline{u}^{\top} H_{n} \beta_{1}}^{\underline{u}^{\top} H_{n}\left(\alpha_{1}+\beta_{1}\right)}\left\{\varphi_{n i}\left(\underline{x}_{k} ; t\right)-\varphi_{n i}\left(\underline{x}_{k} ; t+\underline{u}^{\top} H_{n}\left(\beta_{2}-\beta_{1}\right)\right)\right\} d t \\
& -\int_{\underline{u}^{\top} H_{n}\left(\alpha_{1}+\beta_{1}\right)}^{\underline{u}^{\top} H_{n}\left(\alpha_{2}+\beta_{1}\right)}\left\{\varphi_{n i}\left(\underline{x}_{k} ; t+\underline{u}^{\top} H_{n}\left(\beta_{2}-\beta_{1}\right)\right)-\varphi_{n i}\left(\underline{x}_{k} ; 0\right)\right\} d t \equiv \Delta_{1}+\Delta_{2} .
\end{aligned}
$$

Therefore, $E\left\{Z_{n i}\right\}^{2}=h^{d} \int K^{2}(\underline{u}) f\left(\underline{x}_{k}+h \underline{u}\right) E\left\{\left(\Delta_{1}+\Delta_{2}\right)^{2} \mid X_{i}=\underline{x}_{k}+h \underline{u}\right\} d \underline{u}$. The conclusion is thus obvious observing that by Cauchy inequality and (A.5),

$$
\begin{aligned}
E\left(\Delta_{1}^{2} \mid X_{i}=\underline{x}_{k}+h \underline{u}\right) \leq\left|\underline{u}^{\top} H_{n} \alpha_{1} \underline{u}^{\top} H_{n}\left(\beta_{2}-\beta_{1}\right) \underline{u}^{\top} H_{n} \alpha_{1}\right| \leq 2\left(M_{n}^{(1)}\right)^{2} M_{n}^{(2)} /\left(M^{l} \log n\right), \\
E\left(\Delta_{2}^{2} \mid X_{i}=\underline{x}_{k}+h \underline{u}\right) \leq\left\{\underline{u}^{\top} H_{n}\left(\alpha_{2}-\alpha_{1}\right)\right\}^{2}\left(\left|\underline{u}^{\top} H_{n} \alpha_{2}\right|+\left|\underline{u}^{\top} H_{n} \alpha_{1}\right|+2\left|\underline{u}^{\top} H_{n} \beta_{2}\right|\right) \\
\leq 4\left(M_{n}^{(1)}\right)^{2} M_{n}^{(2)} /\left(M^{l} \log n\right)^{2},
\end{aligned}
$$

where we used the facts that $\left|\alpha_{1}-\alpha_{2}\right| \leq 2 M_{n}^{(1)} /\left(M^{l} \log n\right)$ and $\left|\beta_{1}-\beta_{2}\right| \leq 2 M_{n}^{(2)} /\left(M^{l} \log n\right)$. Therefore, $E\left\{Z_{n i}\right\}^{2}=C h^{d}\left(M_{n}^{(1)}\right)^{2} M_{n}^{(2)} /\left(M^{l} \log n\right)$. As $\left|Z_{n i}\right| \leq C M_{n}^{(1)}$ and $h^{p+1} / M_{n}^{(2)}<\infty$, the rest of the proof can be completed following the proof of Lemma 5.5.

Lemma 5.7 Suppose (A2)- (A6) hold.

$$
\sum_{i=1}^{n} E \Phi_{n i}^{2}+\sum_{i<j}\left|\operatorname{Cov}\left(\Phi_{n i}, \Phi_{n j}\right)\right| \leq C n h^{d}\left(M_{n}^{(1)}\right)^{2} M_{n}^{(2)},
$$

uniformly in $\underline{x} \in \mathcal{D}, \alpha \in B_{n}^{(1)}$ and $\beta \in B_{n}^{(2)}$.

Proof. By Cauchy inequality and (A.5), we have

$$
\begin{aligned}
& E \Phi_{n i}^{2} \\
= & h^{d} \int K^{2}(\underline{u}) E\left[\left\{\int_{\mu(\underline{u})^{\top} H_{n} \beta}^{\mu(\underline{u})^{\top} H_{n}(\alpha+\beta)}\left(\varphi_{n i}(\underline{x} ; t)-\varphi_{n i}(\underline{x} ; 0)\right) d t\right\}^{2} \mid \underline{X}_{i}=\underline{x}+h \underline{u}\right] f(\underline{x}+h \underline{u}) d \underline{u} \\
\leq & h^{d} \int f(\underline{x}+h \underline{u}) K^{2}(\underline{u}) \mu(\underline{u})^{\top} H_{n} \alpha \int_{\underline{u}^{\top} H_{n} \beta}^{\mu(\underline{u})^{\top} H_{n}(\alpha+\beta)} E\left[\left(\varphi_{n i}(\underline{x} ; t)-\varphi_{n i}(\underline{x} ; 0)\right)^{2} \mid \underline{X}_{i}=\underline{x}+h \underline{u}\right] d t d \underline{u} \\
\leq & h^{d} \int K^{2}(\underline{u}) \mu(\underline{u})^{\top} H_{n} \alpha \int_{\mu(\underline{u})^{\top} H_{n} \beta}^{\mu(\underline{u})^{\top} H_{n}(\alpha+\beta)} C|t| d t f(\underline{x}+h \underline{u}) d \underline{u}=O\left\{h^{d}\left(M_{n}^{(1)}\right)^{2} M_{n}^{(2)}\right\},
\end{aligned}
$$

uniformly in $\underline{x} \in \mathcal{D}, \alpha \in B_{n}^{(1)}$ and $\beta \in B_{n}^{(2)}$. (A.53) thus follows from (A.54) and Lemma 5.5. 
Lemma 5.8 Let $(A 3)-(A 6)$ hold. Then

$$
\sup _{\underline{x} \in \mathcal{D}}\left|S_{n p}(\underline{x})-g(\underline{x}) f(\underline{x}) S_{p}\right|=O\left(h+\left(n h^{d} / \log n\right)^{-1 / 2}\right) \text { almost surely. }
$$

Proof. The result is almost the same as Theorem 2 in Masry (1996). Especially if (A.4) holds, then the condition (3.8a) there on the mixing coefficient $\gamma[k]$ is true.

Lemma 5.9 Denote $d_{n 1}=\left(n h^{d}\right)^{1-\lambda_{1}-2 \lambda_{2}}(\log n)^{\lambda_{1}+2 \lambda_{2}}$ and let $\lambda_{1}$ and $B_{n}^{(i)}, i=1,2$, be as in Lemma 5.1. Suppose that $(A 1)-(A 5)$ and (A.2) hold. Then there is a constant $C>0$ such that for each $M>0$ and all large $n$,

$$
\sup _{\substack { x \in \mathcal{D} \\
\begin{subarray}{c}{\alpha \in B_{n}^{(1)} \\
\beta \in B_{n}^{(2)}{ x \in \mathcal { D } \\
\begin{subarray} { c } { \alpha \in B _ { n } ^ { ( 1 ) } \\
\beta \in B _ { n } ^ { ( 2 ) } } }\end{subarray}}\left|\sum_{i=1}^{n} E \Phi_{n i}(\underline{x} ; \alpha, \beta)-\frac{n h^{d}}{2}\left(H_{n} \alpha\right)^{\top} S_{n p}(\underline{x}) H_{n}(\alpha+2 \beta)\right| \leq C M^{3 / 2} d_{n 1} .
$$

Proof. Recall that $G(t, \underline{u})=E(\varphi(Y ; t) \mid \underline{X}=\underline{u})$,

$$
\begin{aligned}
E \Phi_{n i}(\underline{x} ; \alpha, \beta)= & h^{d} \int K(\underline{u}) f(\underline{x}+h \underline{u}) d \underline{u} \times \int_{\mu(\underline{u})^{\top} H_{n} \beta}^{\mu(\underline{u})^{\top} H_{n}(\alpha+\beta)} \\
& \left\{G\left(t+\mu(\underline{u})^{\top} H_{n} \beta_{p}(\underline{x}), \underline{x}+h \underline{u}\right)-G\left(\mu(\underline{u})^{\top} H_{n} \beta_{p}(\underline{x}), \underline{x}+h \underline{u}\right)\right\} d t .
\end{aligned}
$$

By $(A 3)$ and $(A 5)$, we have

$$
\begin{gathered}
G\left(t+\mu(\underline{u})^{\top} H_{n} \beta_{p}(\underline{x}), \underline{x}+h \underline{u}\right)-G\left(\mu(\underline{u})^{\top} H_{n} \beta_{p}(\underline{x}), \underline{x}+h \underline{u}\right) \\
=t G_{1}\left(\mu(\underline{u})^{\top} H_{n} \beta_{p}(\underline{x}), \underline{x}+h \underline{u}\right)+\frac{t^{2}}{2} G_{2}\left(\xi_{n}(t, \underline{u} ; \underline{x}), \underline{x}+h \underline{u}\right), \\
G_{1}\left(\mu(\underline{u})^{\top} H_{n} \beta_{p}(\underline{x}), \underline{x}+h \underline{u}\right)=g(\underline{x}+h \underline{u})+O\left(h^{p+1}\right),
\end{gathered}
$$

where $\xi_{n}(t, \underline{u} ; \underline{x})$ falls between $\mu(\underline{u})^{\top} H_{n} \beta_{p}(\underline{x})$ and $t+\mu(\underline{u})^{\top} H_{n} \beta_{p}(\underline{x})$, and the term $O\left(h^{p+1}\right)$ is uniform in $\underline{x} \in \mathcal{D}$. Therefore, the inner integral in (A.55) is given by

$$
\frac{1}{2} g(\underline{x}+h \underline{u})\left(H_{n} \alpha\right)^{\top} \mu(\underline{u}) \mu(\underline{u})^{\top} H_{n}(\alpha+2 \beta)+O\left\{M^{3 / 2}\left(\frac{\log n}{n h^{d}}\right)^{\lambda_{1}+2 \lambda_{2}}\right\}
$$

uniformly in $\underline{x} \in \mathcal{D}$, where we have used the fact that $n h^{d+(p+1) / \lambda_{2}} / \log n<\infty$. By the definition of $S_{n p}(\underline{x})$, the proof is thus completed. 
Lemma 5.10 Under conditions in Theorem 3.2, we have

$$
\sup _{\underline{x} \in \mathcal{D}}\left|\frac{1}{n h^{d}} W_{p} S_{n p}^{-1}(\underline{x}) H_{n}^{-1} \sum_{i=1}^{n} K_{h}\left(\underline{X}_{i}-\underline{x}\right) \varphi\left(\varepsilon_{i}\right) \mu\left(\underline{X}_{i}-\underline{x}\right)\right|=O\left\{\left(\frac{\log n}{n h^{d}}\right)^{1 / 2}\right\} \text { almost surely. }
$$

Proof. Note that under conditions Theorem 3.2, the assumptions imposed by Masry (1996) in Theorem 5 hold. Specifically, (4.5) there follows from (A.2), and (4.7b) there from (A.4). Therefore, mimicking the proof lines there, we can show that

$$
\sup _{\underline{x} \in \mathcal{D}}\left|\frac{1}{n h^{d}} H_{n}^{-1} \sum_{i=1}^{n} K_{h}\left(\underline{X}_{i}-\underline{x}\right) \varphi\left(\varepsilon_{i}\right) \mu\left(\underline{X}_{i}-\underline{x}\right)\right|=O\left\{\left(\frac{\log n}{n h^{d}}\right)^{1 / 2}\right\}
$$

which together with Lemma 5.8 yields the desired results. 